\title{
Connexin 30 Deficiency Attenuates Chronic but Not Acute Phases of Experimental Autoimmune Encephalomyelitis Through Induction of Neuroprotective Microglia
}

\author{
Mei Fang ${ }^{1}$, Ryo Yamasaki ${ }^{1}$, Guangrui Li $^{1}$, Katsuhisa Masaki ${ }^{1}$, Hiroo Yamaguchi ${ }^{1}$, \\ Atsushi Fujita ${ }^{1}$, Noriko Isobe ${ }^{1,2}$ and Jun-ichi Kira ${ }^{1 *}$ \\ ${ }^{1}$ Department of Neurology, Neurological Institute, Graduate School of Medical Sciences, Kyushu University, Fukuoka, Japan, \\ ${ }^{2}$ Department of Neurological Therapeutics, Neurological Institute, Graduate School of Medical Sciences, Kyushu University, \\ Fukuoka, Japan
}

Glial connexins (Cxs) form gap junction channels through which a pan-glial network plays key roles in maintaining homeostasis of the central nervous system (CNS). In multiple sclerosis (MS) and its animal model, experimental autoimmune encephalomyelitis (EAE), expression of astrocytic $\mathrm{C} \times 43$ is lost in acute lesions but upregulated in chronic plaques, while astrocytic Cx30 is very low in normal white matter and changes in its expression have not been convincingly shown. In $\mathrm{C} \times 30$ or $\mathrm{C} \times 43$ single knockout (KO) mice and even in Cx30/Cx43 double KO mice, acute EAE is unaltered. However, the effects of Cx30/Cx43 deficiency on chronic EAE remains to be elucidated. We aimed to clarify the roles of $\mathrm{C} \times 30$ in chronic neuroinflammation by studying EAE induced by myelin oligodendrocyte glycoprotein peptide 35-55 in Cx30 KO mice. We found that Cx30 deficiency improved the clinical symptoms and demyelination of chronic but not acute EAE without influencing $\mathrm{CD}^{+} \mathrm{T}$ cell infiltration. Furthermore, increased ramified microglia in the naïve state and induced earlier and stronger microglial activation in the acute and chronic phases of EAE was observed. These activated microglia had an anti-inflammatory phenotype, as shown by the upregulation of arginase- 1 and brain-derived neurotrophic factor and the downregulation of nitric oxide synthase 2. In the naive state, Cx30 deficiency induced modest enlargement of astrocytic processes in the spinal cord gray matter and a partial reduction of $\mathrm{C} \times 43$ expression in the spinal cord white matter. These astrocytes in $\mathrm{C} \times 30 \mathrm{KO}$ mice showed earlier and stronger activation during the acute phase of EAE, with upregulated A2 astrocyte markers and a significant decrease in Cx43 in the chronic phases. Spinal cord neurons and axons were more preserved in Cx30 KO mice than in littermates in the chronic phase of EAE. These findings suggest that $\mathrm{Cx} 30$ deficiency increased ramified microglia in the CNS in the naive state and improved chronic EAE through redirecting microglia toward an anti-inflammatory phenotype, suggesting a hitherto unknown critical role of astrocytic Cx30 in regulating microglial number and functional state.

Keywords: astrocyte, chronic neuroinflammation, connexin, experimental autoimmune encephalomyelitis, microglia, multiple sclerosis 


\section{INTRODUCTION}

Multiple sclerosis (MS) is an inflammatory demyelinating disease of the central nervous system (CNS) (1). It initially presents as relapsing remitting MS (RRMS) but later evolves into secondary progressive MS (SPMS) in $\sim 20 \%$ of patients, even after disease-modifying therapies (DMTs) are introduced $(2,3)$. Most DMTs, mainly targeting the peripheral immune system, can effectively reduce relapses in RRMS; however, they are of little benefit for chronic progression in SPMS (4-6). Thus, chronic progression in MS is currently a matter of concern for research and drug development. Recently, siponimod (7) and ozanimod (8), new functional antagonists of sphingosine1-phosphate receptor 1 (S1P1), were reported to be effective for preventing disability progression in SPMS. These drugs may directly act on glial cells harboring S1P1 such as microglia and astroglia, in addition to their inhibitory effects on lymphocyte egress from the secondary lymphoid organs (9-11). In chronic MS lesions, persistent demyelination with varying degrees of remyelination and neuroaxonal degeneration are accompanied by the presence of activated microglia but few $\mathrm{T}$ cells (12, 13), suggesting a key role of microglia, which are not targeted by the peripherally acting DMTs, in chronic inflammation in SPMS.

Connexins (Cxs) form gap junction (GJ) channels, which allow the intercellular exchange of ions and secondary messengers (14). In the CNS, astrocytes express Cx43, Cx30, and Cx26, while oligodendrocytes express Cx47, Cx32, and Cx29 (15-17). These Cxs constitute a pan-glial network through GJ channels and play key roles in maintaining CNS homeostasis (18-20). We and others have reported dynamic changes of glial Cxs in MS and Balós concentric sclerosis lesions (21-23). Oligodendrocytic $\mathrm{Cx} 47$ and $\mathrm{Cx} 32$ are persistently lost in acute and chronic MS plaques, while astrocytic Cx43 is lost in acute lesions and then upregulated in chronic astrogliotic plaques (22-24). Similar changes in $\mathrm{Cx} 47, \mathrm{Cx} 32$, and $\mathrm{Cx} 43$ were also observed in acute and chronic experimental autoimmune encephalomyelitis (EAE), an animal model of MS (25-28). These findings suggest the involvement of glial Cxs in inflammatory demyelination.

Consistent with this notion, oligodendrocytic Cx32 knockout (KO) mice developed aggravated acute and chronic EAE, with increased demyelination despite a similar degree of inflammation upon immunization with myelin oligodendrocyte glycoprotein (MOG), compared with wild type (WT) mice (25). By contrast, in astrocytic $\mathrm{Cx} 30$ or $\mathrm{Cx} 43$ single $\mathrm{KO}$ mice and even in $\mathrm{Cx} 30 / \mathrm{Cx} 43$ double KO mice, acute EAE was unaltered (29). However, it remains to be elucidated whether a deficiency in $\mathrm{Cx} 30$ or $\mathrm{Cx} 43$ influences chronic EAE.

Because the expression level of astrocytic Cx30 is very low in normal white matter, changes of $\mathrm{Cx} 30$ in MS or EAE lesions have not been well demonstrated (22, 23, 30). Cx43 exists in both mature and immature astrocytes, while Cx30 is expressed only in mature astrocytes (31-33), thus gliotic scar astrocytes show an upregulation of $\mathrm{Cx} 43$ but no detectable changes of Cx30 (23). Similarly, cultured astrocytes express detectable levels of $\mathrm{Cx} 43$ but not $\mathrm{Cx} 30$, although they can express Cx30 after very long term culture (33, 34). These features of Cx30 make it difficult to study its dynamics and roles in inflammatory demyelination, and therefore there have been few studies of Cx30 in EAE. However, the non-channel functions of Cxs have recently gained increasing attention: Cx30 can change astrocyte morphology, thereby modulating astrocyte functions such as synaptic transmission (35). Cxs also inhibits DNA synthesis, which affects the gene expression network $(36,37)$.

In the present study, we aimed to clarify the roles of $\mathrm{Cx} 30$ in chronic neuroinflammation by studying chronic EAE in Cx30 KO mice. Here, we report Cx30 deficiency induces antiinflammatory microglia and improves clinical symptoms and demyelination of chronic but not acute EAE.

\section{MATERIALS AND METHODS}

\section{Ethics Statement}

The experimental procedures were designed to minimize the number of animals used as well as animal suffering. All animal experiments were carried out according to the guidelines for proper conduct of animal experiments published by the Science Council of Japan and the ARRIVE (Animal Research: Reporting of in vivo Experiments) guidelines for animal research. Ethical approval for the study was granted by the Animal Care and Use Committee of Kyushu University (\#A29-146-3).

\section{Animals and Genotyping}

Twelve-to-sixteen-week-old female $\mathrm{Cx} 30 \mathrm{KO}$ mice were used in this study. Cx30 KO mice (38) that had been backcrossed to $\mathrm{C} 57 \mathrm{BL} / 6 \mathrm{~J}$ at the archiving center were purchased from the European Mouse Mutant Archive. C57BL/6 mice were purchased from KBT Oriental (Tosu, Japan). All mice were bred and maintained under specific pathogen free conditions in the Center of Biological Research, Graduate School of Medical Sciences, Kyushu University. The Cx30 KO mice were genotyped by PCR of DNA obtained from tail biopsies. Primer pairs for detecting Cx30 KO were Cx30 KO-1 (LACZ e Neo): 5'-GGT ACC TTC TAC TAA TTA GCTTGG-3'; Cx30 KO2 (LACZ e Neo): 5'-AGG TGG TAC CCA TTG TAG AGG AAG-3'; and Cx30 KO-3 (LACZ e Neo): 5'-AGC GAG TAA CAA CCC GTC GGA TTC-3'. The $\mathrm{Cx} 30 \mathrm{KO}$ and WT littermate DNA products were 460 and 544 bps in size, respectively. Cx30 KO mice and their littermates were principally used for the animal experiments, unless otherwise specified.

\section{Induction and Clinical Evaluation of EAE}

EAE was induced by immunization of mice with $200 \mu \mathrm{g}$ of $\mathrm{MOG}_{35-55}$ peptide (TS-M704-P; MBL, Nagoya, Japan) in 50 $\mu l$ phosphate buffered saline (PBS) emulsified in an equal volume of complete Freund's adjuvant (CFA) containing 1 $\mathrm{mg} / \mathrm{ml}$ Mycobacterium tuberculosis H37RA (\#231131; BD Difco, Lawrence, KS, USA), followed by intraperitoneal injections of 500 ng pertussis toxin (\# 180-A1; List Biological Laboratories Inc., Campbell, CA, USA) on days 0 and 2. Mice were examined daily for signs of EAE and scored as follows: 0 , no disease; 1, limp tail; 2 , abnormal gait and hind limb weakness (shaking); 2.5 , paralysis 
of one hind limb; 3, paralysis of two hind limbs; 3.5, ascending paralysis (able to move around); 4 , tetraplegia; 5 , moribund.

\section{Tissue Preparation}

Animals were deeply anesthetized by isoflurane (Pfizer Japan Inc., Tokyo, Japan), and perfused transcardially with PBS and then with $4 \%$ paraformaldehyde (PFA) in $0.1 \mathrm{M}$ PBS. Spinal cords, brains and optic nerves were carefully dissected. The tissues were fixed overnight in cold $4 \% \mathrm{PFA}$ at $4^{\circ} \mathrm{C}$, then processed into paraffin sections $(5 \mu \mathrm{m})$. For frozen sections $(20 \mu \mathrm{m})$, spinal cords were harvested and fixed overnight in 4\% PFA using the same protocol as above and sequentially displaced with 15 and $30 \%$ sucrose in PBS for $24 \mathrm{~h}$ each at $4^{\circ} \mathrm{C}$. The resulting tissues were embedded in Tissue-Tek O.C.T. Compound (4583, Sakura Finetek, Torrance, CA, USA) and stored at $-80^{\circ} \mathrm{C}$.

\section{Histopathological and Immunohistochemical Analyses}

Paraffin-embedded sections of spinal cord were stained with hematoxylin and eosin (HE). Paraffin-embedded sections of optic nerves were subjected to immunohistochemistry using an indirect immunoperoxidase method. After deparaffinization, endogenous peroxidase was quenched with $0.3 \%$ hydrogen peroxide in absolute methanol for $30 \mathrm{~min}$. The sections were permeabilized with $0.1 \%$ Triton in PBS (PBS-T) for $10 \mathrm{~min}$, washed using Tris- $\mathrm{HCl}$ for $5 \mathrm{~min}$, dipped in $10 \mathrm{mM}$ citrate buffer, and then autoclaved $\left(120^{\circ} \mathrm{C}, 10 \mathrm{~min}\right)$. All sections were cooled to room temperature and incubated with anti-brain-derived neurotrophic factor (BDNF) antibodies overnight at $4^{\circ} \mathrm{C}$ (Supplementary Table 1). The next day, after rinsing, sections were labeled with either a streptavidinbiotin complex or an enhanced indirect immunoperoxidase method using Envision (K4003, Dako, Glostrup, Denmark); 3,3-diaminobenzidine tetrahydrochloride (DAB; D5637, Sigma-Aldrich, Tokyo, Japan) was used for the DAB color reaction. Finally, sections were counterstained with hematoxylin.

\section{Confocal Microscope \\ Immunofluorescence Analysis}

Paraffin sections of brain and optic nerves were deparaffinized in xylene and rehydrated through ethanol. After washing and autoclaving, sections were incubated with anti-arginasel, anti-nitric oxide synthase 2 (NOS2), anti-Iba-1, anti-glial fibrillary acidic protein (GFAP), anti-Cx43, anti-Cx30, anti-myelin basic protein (MBP), anti-NeuN, purified antineurofilament H (NF-H) (SMI-31), and anti-IL-34 antibodies (Supplementary Table 1) overnight at $4^{\circ} \mathrm{C}$. The following day, the sections were washed, incubated with Alexa Fluor 488- or 546-conjugated secondary antibodies (1:1,000; Thermo Fisher, Rockford, IL, USA) and 4',6-diamidino-2-phenylindole (DAPI; Sigma-Aldrich, Tokyo, Japan) overnight at $4^{\circ} \mathrm{C}$, then dehydrated and sealed with Permafluor (\#TA-030-FM; Thermo Scientific, Fremont, CA, USA). The frozen sections of spinal cords were cut at $20 \mu \mathrm{m}$ with a cryostat microtome (Leica CM 1850, Leica Microsystems GmbH, Wetzlar, Germany) and floated in PBS-T. The sections were washed 3 times in PBS-T, blocked with $10 \%$ normal goat serum in PBS for $2 \mathrm{~h}$, then incubated overnight at $4^{\circ} \mathrm{C}$ with anti-arginase1, anti-NOS2, anti-Cx30, anti-Cx43, anti-Iba-1, anti-GFAP, anti-CD45, anti-CD3, antiC3, and anti-CD169 antibodies (Supplementary Table 1). The sections were also treated with anti-S100a10 antibody in the same way but without blocking by normal goat serum (Supplementary Table 1). After rinsing the next day, the sections were incubated with Alexa Fluor 488- or 546-conjugated secondary antibodies (1:1,000; Thermo Fisher) or FluoroMyelin Red Fluorescent Myelin Stain (1:1,000; \#F34652; Thermo Fisher) and DAPI overnight at $4{ }^{\circ} \mathrm{C}$, then washed in PBS-T and sealed with Permafluor. Immunofluorescence was captured by a confocal laser microscope (Nikon A1; Nikon, Tokyo, Japan), equipped with 405,488 , and $561 \mathrm{~nm}$ laser lines, at the same magnification, laser intensity, gain, offset values, and pinhole settings. Quantification of immunofluorescence was performed using ImageJ version 1.6.0_24 (Windows version of NIH Image; downloaded from https://imagej.nih.gov/ij/download.html) on three-to-five lumbar spinal cord sections for each animal in each group.

\section{Quantification of Myelin Density and Cell Infiltration in the Spinal Cord, Brain, and Optic Nerve}

For the quantification of GFAP, Iba-I, Cx30, Cx43, CD3, CD169, CD45, S100A10, C3, BDNF, NOS2, and IL-34, fluorescent images from the anterior part of the lumbar spinal cord, cerebellum, cerebrum, and optic nerve were analyzed (ImageJ version 1.6.0_24) using the area fraction technique as previously described $(39,40)$. Briefly, identical microscope settings were applied to all photographs from each experiment and images from the same areas were acquired. Images were de-noised and set to the same threshold baseline across experimental groups for each antibody to measure the area of cellular staining, instead of cell density measurement or cell number counting, because most infiltrating cells were focally clustered. For the quantification of myelin and MBP immunostaining results, whole spinal cord images were captured under the microscope and separated into anterior or posterior parts for analysis. SMI-31 immunostaining images were captured under the microscope and spinal cord anterior white matter areas were used for analysis. Image analysis was performed using ImageJ software. Mean pixel intensity values were compared between genotypes (41). For the quantitative analyses of NeuNpositive cells, transverse sections of the spinal cord were divided into the left and right regions by a vertical line through the central canal. The size of each microscopic field was 1.6384 $\mathrm{mm}^{2}$. The left or right positive cell region areas (0.33-0.44 $\mathrm{mm}^{2}$ ) were calculated automatically by ImageJ. NeuN-positive cells were counted manually and used to calculate the cell density (neurons $/ \mathrm{mm}^{2}$ ) (42). The investigator performing the analysis was blinded to the genotypes. All assessments were made from three-to-five sections per mouse ( $n=3$ to 8 mice in each group). In the quantification graph, the mean value of three-to-five sections was used as scatter dots to represent each mouse. 


\section{Immunocolocalization Analysis}

We delineated the same areas of focus in the spinal cord white matter, optic nerve, and arbor vitae of the cerebellum in all samples to be analyzed. Colocalization of arginase-1 and Iba-1 was expressed as a Pearson's correlation coefficient and the intensity correlation analysis plugin of Image J was used (43). Pearson's correlation values range from 1 to -1 , with 1 representing complete positive correlation and -1 a negative correlation, with zero representing no correlation. All quantifications were obtained from a minimum of three sections from the spinal cord, optic nerve, cerebellum, and cerebrum per mouse.

\section{Microglial Circularity Analysis}

ImageJ was used to automatically calculate the circularity of microglial cells (circularity $=4 \pi \mathrm{S} / \mathrm{L} 2$ ). Cells with circularity close to 1 were regarded as having a morphology close to round, indicating an activated state $(44,45)$.

\section{Microglial Cell Isolation and Flow Cytometry}

Brains and spinal cords were harvested and homogenized. Mononuclear cells were separated with a 30 and 70\% Percoll (GE Healthcare, Tokyo, Japan) gradient as previously described (46). Cells were stained with anti-CD45-PerCP and anti-CD11bPE/Cy7 antibodies, sorted and analyzed on a SH800 Cell Sorter (Sony Corporation, Tokyo, Japan).

\section{Gene Expression Microarray}

Total RNA was isolated from cells using an RNeasy Mini Kit (Qiagen) according to the manufacturer's instructions. RNA samples were quantified by an ND-1000 spectrophotometer (NanoDrop Technologies, Wilmington, DE, USA) and the quality was confirmed with a 2200 TapeStation (Agilent technologies, Santa Clara, CA, USA). Total RNA (2 ng) was amplified, labeled using a GeneChip ${ }^{\circledR}$ WT Pico Kit, and hybridized to an Affymetrix GeneChip ${ }^{\circledR}$ Mouse Transcriptome Array 1.0 according to the manufacturer's instructions (Affymetrix, Santa Clara, CA, USA). All hybridized microarrays were scanned by an Affymetrix scanner. Relative hybridization intensities and background hybridization values were calculated using the Affymetrix Expression Console ${ }^{\circledR}$. These gene array assay results were uploaded to the gene expression omnibus repository (accession number is GSE68202) in the National Center for Biotechnology Information homepage (https://www. ncbi.nlm.nih.gov/geo/query/acc.cgi?acc=GSE112621).

\section{Data Analysis and Filter Criteria}

The raw signal intensities of all samples were normalized by a quantile algorithm with Affymetrix ${ }^{\circledR}$ Power Tool version 1.15.0 software. To identify upregulated or downregulated genes, we calculated Z-scores [Z] and ratios (non-log scaled foldchange) from the normalized signal intensities of each probe for comparison between control and experiment samples. Then, we established criteria for regulated genes: upregulated genes had a $\mathrm{Z}$-score $\geq 2.0$ and ratio $\geq 1.5$-fold, and downregulated genes had a $\mathrm{Z}$-score $\leq-2.0$ and ratio $\leq 0.66$. Gene set enrichment analysis
(47, 48) (GSEA; www.broadinstitute.org/gsea) was performed to investigate deviations of particular gene sets (e.g., Antiinflammatory set, Pro-inflammatory set; set S) according to a previous report $(48,49)$. Briefly, after we obtained expression data sets for each study group, we calculated an enrichment score (ES) that reflected the degree to which a set "S" was overrepresented at the extremes (top or bottom) of the entire ranked list "L." The score was calculated by walking down the list $\mathrm{L}$ and increasing a running-sum statistic when we encountered a gene in $\mathrm{S}$. The ES is the maximum deviation from zero encountered in the random walk. After the estimation of statistical significance of ES, we controlled the proportion of false positives by calculating the false discovery rate (FDR). When the normalized $p$-value was $<0.05$ and the FDR was $<0.25$, the ES was considered significant.

\section{Statistical Analysis}

Data are expressed as the mean \pm standard error of mean (S.E.M.). The area under curve (AUC) of the overall disease severity was calculated for each mouse to compare the disease course of WT and KO mice using the non-parametric MannWhitney $U$-test (50) Here, acute (onset to day 24) and chronic phases (day 25 and thereafter) were separately analyzed. The postimmunization date when WT groups reached a peak score of 2 or higher was identified as the "peak" (51). The incidence, day of onset, and peak clinical score of EAE were compared using the unpaired $t$-test with Welch's correction. In EAE experiments, mice that died before the intended day of sacrifice were excluded from statistical analyses. Cell percentages and histological data were assessed by the unpaired $t$-test with Welch's correction, two-way ANOVA, or one-way ANOVA. A $p$-value $<0.05$ was considered statistically significant. Analyses were performed using Graph Pad Prism 7.0 software (Graph Pad, La Jolla, CA, USA).

\section{RESULTS}

\section{Cx30 Deficiency Induces Modest Morphological Changes and Cx43 Reduction in Spinal Cord Astrocytes but No Changes in Myelin Density}

In WT littermate mice in the naïve state, Cx30 was expressed predominantly on astrocytes in the gray matter of the spinal cord, cerebellum and cerebrum, while Cx30 expression was very low in white matter astrocytes, including the optic nerve (Figure 1A; Supplementary Figure 1), which is consistent with our previous study in humans (22). By contrast, Cx30 was completely absent in Cx30 KO mice (Figures 1A-C; Supplementary Figure 1). GFAP immunostaining revealed neither morphological nor quantitative changes in $\mathrm{GFAP}^{+}$astrocytes in the white matter between WT and $\mathrm{Cx} 30 \mathrm{KO}$ mice, whereas $\mathrm{GFAP}^{+}$astrocytes in the gray matter had thicker processes and showed a tendency to be increased in Cx30 KO mice than in WT mice ( $p=0.0575$, Figures 1A,D,E).

Because astrocytic $\mathrm{Cx} 30$ and $\mathrm{Cx} 43$ have similar functions and partly overlapping permeation profiles (15), we examined whether $\mathrm{Cx} 43$ was upregulated to compensate for the lack of $\mathrm{Cx} 30$ in $\mathrm{Cx} 30 \mathrm{KO}$ mice. GFAP and $\mathrm{Cx} 43$ double immunostaining 


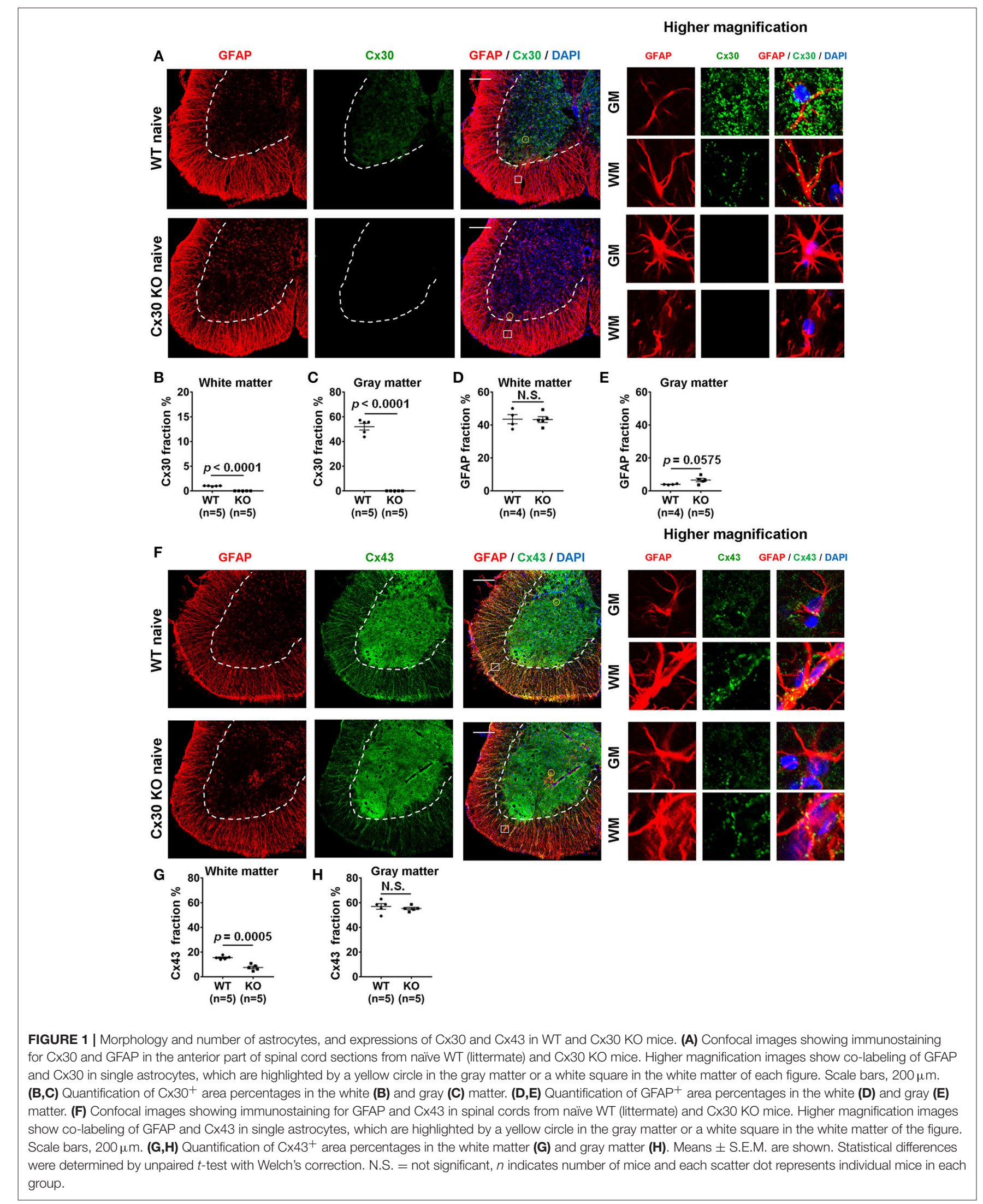


revealed that $\mathrm{Cx} 43$ was more abundant in the gray matter than in the white matter of the spinal cord in both WT and Cx30 KO mice (Figures 1F-H). There was no significant difference in $\mathrm{Cx} 43$ immunoreactivity in the spinal cord gray matter between WT and $\mathrm{Cx} 30 \mathrm{KO}$ mice, but $\mathrm{Cx} 43$ levels were significantly reduced in the spinal cord white matter of $\mathrm{Cx} 30 \mathrm{KO}$ mice compared with WT mice ( $p=0.0005$; Figures $\mathbf{1 F}-\mathbf{H})$. These findings are consistent with the observation that $\mathrm{Cx} 30$ but not $\mathrm{Cx} 43$ can compensate for other Cxs in CNS tissues (52). This suggests that Cx30 deficiency causes modest enlargement of astrocytic processes in the spinal gray matter and a partial reduction of $\mathrm{Cx} 43$ expression in spinal white matter astrocytes. However, myelin density did not differ significantly between Cx30 KO mice and WT littermates in the naive state by Fluoromyelin staining or MBP immunostaining (Supplementary Figures 2A-D).

\section{Cx30 Deficiency Increases the Numbers of Ramified Microglia}

Unexpectedly, Cx30 KO mice showed an apparent increase in the numbers of $\mathrm{Iba}_{-1}^{+}$microglia in the spinal cord gray matter, optic nerve, cerebellum, and cerebrum compared with WT littermate mice $(p=0.0055, p=0.0274, p=0.0015$, and $p=0.0012$, respectively), but not in the spinal cord white matter (Figures 2A-E). Morphologically, microglia in $\mathrm{Cx} 30 \mathrm{KO}$ mice had thin soma and delicate radially-projecting processes (Figure 2A inset), indicating that these microglia were in a resting state (ramified phenotype). There were no significant differences in the microglia circularity index between $\mathrm{Cx} 30 \mathrm{KO}$ and WT mice in the spinal cord gray and white matter, optic nerve, cerebrum, and cerebellum (Figures 2B-E). Flow cytometric analyses demonstrated that numbers of $\mathrm{CD} 45^{\mathrm{dim}} \mathrm{CD} 11 \mathrm{~b}^{+}$microglia in isolated viable cells from the brain were significantly increased in $\mathrm{Cx} 30 \mathrm{KO}$ mice compared with WT littermate mice ( $p=0.0025$; Figures 2F,G).

To further characterize the microglial phenotype in Cx30 KO mice, gene expression profiles were analyzed by RNA microarray using microglia isolated from the spinal cords and brains of naïve WT and Cx30 KO mice. Microglia from Cx30 KO mice showed similar expression levels of antiinflammatory and pro-inflammatory genes to WT microglia in both the spinal cord and brain (Table $\mathbf{1}$ and Figure 3A). GSEA analysis revealed similar gene enrichments in the spinal cord and brain between naive WT and KO mice (Table 1 and Figures 3B-E). We also performed GSEA analysis to characterize the expression profiles of cytokines/chemokines, complement, alarmin, reactive oxygen species (ROS), MHC, and tumor genes. Among them, Cx30 KO microglia from the naïve spinal cord but not brain demonstrated significantly lower expression levels of cytokines/chemokines, alarmin, MHC, and tumor genes, indicating a less reactive state to inflammatory insults (Table 1 and Supplementary Figures $\mathbf{3 A}, \mathbf{C}$ ). These findings indicate that the increase in microglia was widespread in the CNS of naïve Cx30 KO mice compared with WT mice; these microglial were not activated but rather in a resting state, with a ramified morphology and low cytokine/chemokine, alarmin, MHC, and tumor gene production.

\section{Cx30 Deficiency Attenuates the Clinical Severity and Demyelination of Chronic but Not Acute EAE Without Influencing T Cell Infiltration}

Cx30 KO mice did not show any significant differences in the incidence, onset day, and clinical severity (peak score and acute phase AUC from Day 9 to 24) of acute EAE compared with WT mice, in agreement with a previous study (29) (Figure 4A). By contrast, clinical severity in the chronic EAE phase (chronic phase AUC from Day 25 to 59) was significantly attenuated in $\mathrm{Cx} 30 \mathrm{KO}$ mice compared with WT mice. HE staining showed that the infiltration of inflammatory cells into the spinal cord was markedly reduced in Cx30 $\mathrm{KO}$ mice compared with WT mice in the chronic EAE phase (Figure 4B). Moreover, the extent of demyelination was significantly decreased in the chronic but not acute phase of EAE in Cx30 KO mice compared with WT mice, in both the anterior and posterior parts of the spinal cord $(p=0.0031$ and $p=0.002$, respectively, by Fluoromyelin staining; and $p=0.0328$ and $p$ $=0.0167$, respectively, by MBP immunostaining; Figures $4 \mathrm{C}-\mathrm{E}$, Supplementary Figures 2E,F). CD $45^{+}$immunocytes and $\mathrm{CD}^{+}$ $\mathrm{T}$ cells were not significantly different between Cx30 KO and WT mice in either acute or chronic phases, although $\mathrm{CD} 45^{+}$cells tended to be increased in $\mathrm{Cx} 30 \mathrm{KO}$ mice compared with WT mice at acute phase $(p=0.0622$; Figures $4 \mathbf{C}, \mathbf{D}, \mathbf{F})$. We performed CD169 immunostaining to discriminate peripheral blood-borne macrophages from microglia and other immune cells, and found that $\mathrm{CD} 169^{+}$macrophages were significantly lower in $\mathrm{Cx} 30 \mathrm{KO}$ mice compared with WT mice in the chronic phase $(p=0.0336)$, but not the acute phase (Figures 5A-C).

\section{Microglia in Cx30 KO Mice Are Widespread and Highly Activated in the Chronic Phase of EAE}

Intriguingly, Iba- $1^{+}$microglial cell numbers were consistently greater in both the white and gray matter of the spinal cord in Cx30 KO mice compared with WT mice in the acute and chronic phases (white matter, $p=0.0106$ on Day 13 and $p=$ 0.0274 on Day 59; gray matter, $p=0.0483$ on Day 13 and $p$ $=0.0111$ on Day 59; Figures 5D,E,G). The above-mentioned increased tendency of CD $45^{+}$cells in the acute phase in Cx30 $\mathrm{KO}$ mice might be explained by the earlier and stronger increase of Iba- $1^{+}$cells compared with WT mice. In the spinal cord white matter, microglial circularity was significantly greater in Cx30 KO mice than in WT mice in both the acute $(p=0.045)$ and chronic phases $(p=0.0418$; Figure 5H). This increase in Iba- $1^{+}$cells in the chronic phase of EAE was also observed in the optic nerve $(p=0.0067)$ and cerebellum $(p=0.0312)$ of Cx30 KO mice (Supplementary Figures 4A,B,D,E). In the cerebellum, microglial circularity was significantly greater $(p=$ 0.0121) in Cx30 KO mice than in WT mice but there was no significant increase of microglial circularity in the optic nerve (Supplementary Figures 4C,F). These findings suggest that a widespread increase and activation of Iba- $1^{+}$microglia in inflamed CNS tissues, especially the spinal cord white matter, is a characteristic feature of $\mathrm{Cx} 30 \mathrm{KO}$ mice. 
A

\section{Spinal cord}
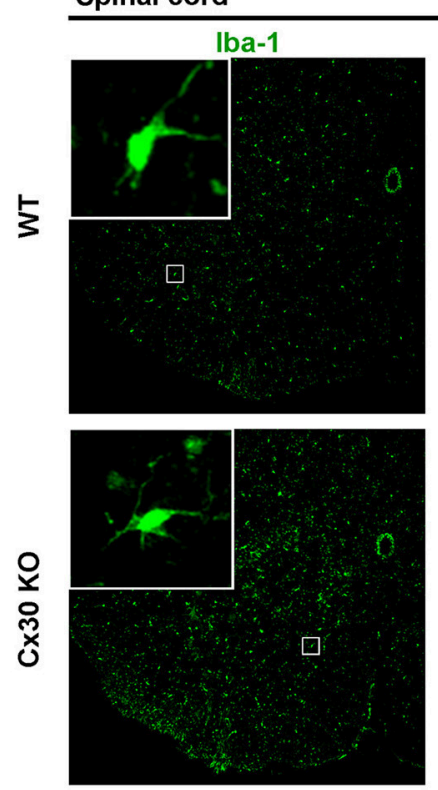

。

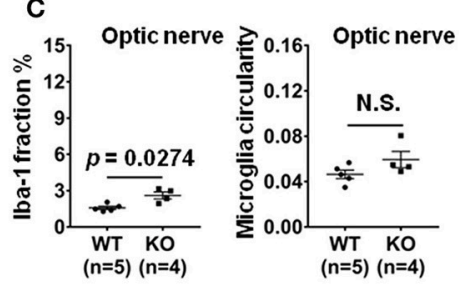

F

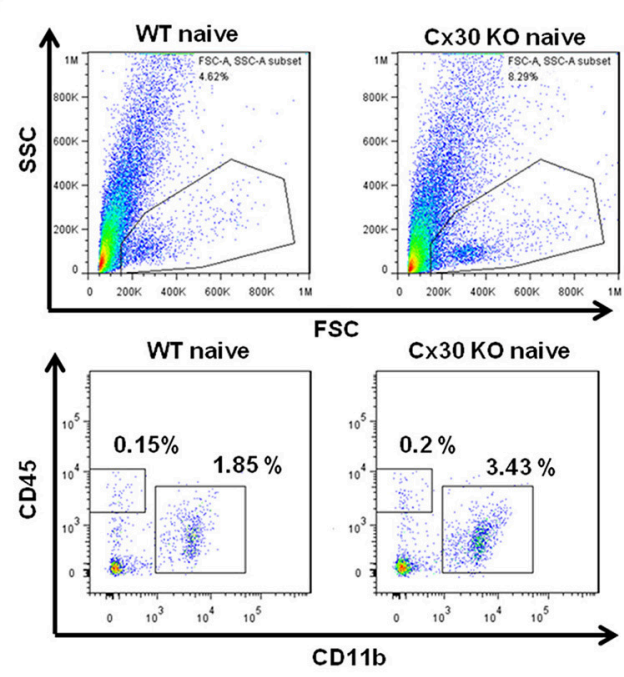

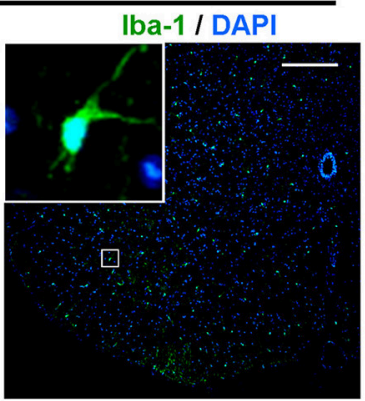

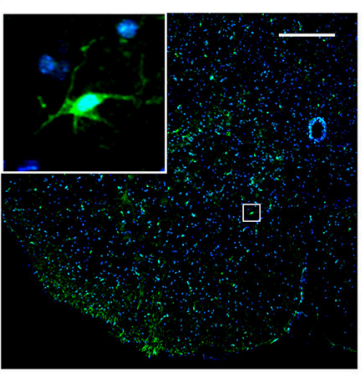

D
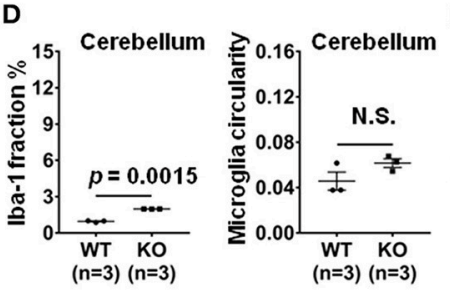

B

Spinal cord

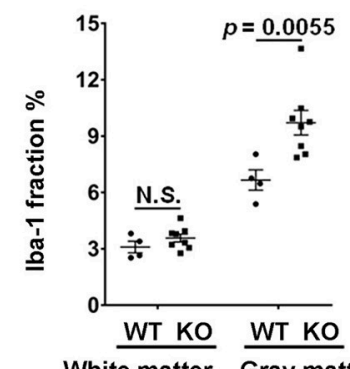

White matter Gray matter

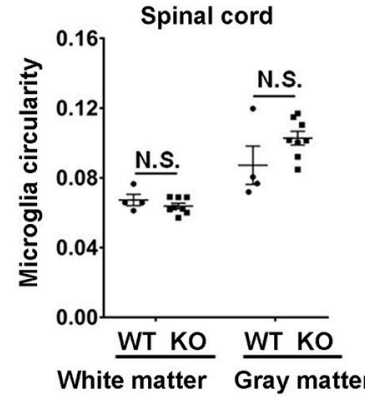

E

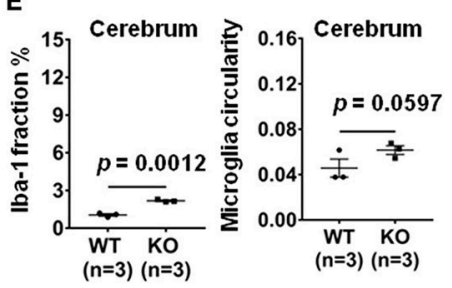

G

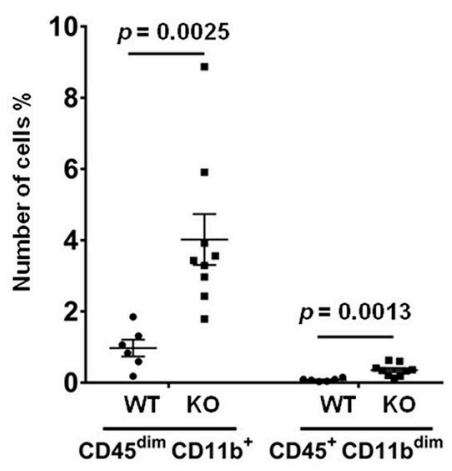

FIGURE 2 | Microglial morphology and numbers in CNS tissues of WT and Cx30 KO mice. (A) Representative images of lba-1 immunostaining of naïve WT (littermate) and Cx30 KO mouse spinal cord. Scale bars, $200 \mu \mathrm{m}$. (B-E) Quantification of lba-1+ cell area fractions and microglia circularity in the white and gray matter of the anterior spinal cord (B), optic nerve (C), cerebellum (D), and cerebrum (E). (F) Gating strategy used to determine viable mononuclear cells for further analysis. In the whole brain suspension, a gate was created on the non-debris population. Inside this population, the microglial cells were gated based on CD45/CD11b intensity. SSC, Side scatter; FSC, Forward scatter. Representative flow cytometric analysis of microglia (CD45 dim $\mathrm{CD}^{\mathrm{fl}} \mathrm{b}^{+}$cells) isolated from WT (littermate) and Cx30 KO mouse brains. Numbers on plots are percentages of double-positive cells among the gated viable cells. (G) Percentages of CD45 dim $\mathrm{CD} 11 \mathrm{~b}^{+}$microglia and $\mathrm{CD} \mathrm{f}^{+}$ $\mathrm{CD} 11 \mathrm{~b}{ }^{\text {dim }}$ cells in the total cell population $\left(3 \times 10^{4}\right)$ isolated from naïve WT (littermate) and Cx30 KO mouse brains. Data are from 6 WT mice and 9 Cx30 KO mice. Means \pm S.E.M. are shown. Statistical differences were determined using the unpaired $t$-test with Welch's correction. N.S. $=$ not significant. $n$ indicates the number of mice and each scatter dot represents individual mice in each group. 
TABLE 1 | Summary of GSEA results.

\begin{tabular}{|c|c|c|c|c|}
\hline \multirow[t]{2}{*}{ Gene category } & Naive spinal cord & EAE spinal cord & Naive brain & EAE brain \\
\hline & WT vs. Cx30 KO & WT vs. Cx30 KO & WT vs. Cx30 KO & WT vs. Cx30 KO \\
\hline Pro-inflammatory & $0.392(\mathrm{WT})$ & 0.007 (WT) & 0.571 (Cx30 KO) & $0.016(\mathrm{WT})$ \\
\hline Anti-inflammatory & $0.083(\mathrm{WT})$ & $0.092(\mathrm{WT})$ & $0.234(\mathrm{WT})$ & $0.450(\mathrm{WT})$ \\
\hline Cytokines/Chemokines & $<0.001$ (WT) & $<0.001$ (WT) & $0.773(\mathrm{WT})$ & $0.252(\mathrm{C} \times 30 \mathrm{KO})$ \\
\hline Complement & 0.997 (Cx30 KO) & 0.001 (WT) & $0.576(\mathrm{WT})$ & 0.348 (C×30 KO) \\
\hline Alarmin & $<0.001(\mathrm{WT})$ & 0.005 (WT) & 0.898 (Cx30 KO) & $0.061(\mathrm{WT})$ \\
\hline ROS & $0.524(\mathrm{WT})$ & 0.005 (WT) & 0.093 (Cx30 KO) & $0.363(\mathrm{C} \times 30 \mathrm{KO})$ \\
\hline $\mathrm{MHC}$ & $<0.001(\mathrm{WT})$ & 0.008 (WT) & 0.173 (WT) & $<0.001(\mathrm{Cx30} \mathrm{KO})$ \\
\hline Tumor & $<0.001$ (WT) & $<0.001$ (WT) & 0.967 (Cx30 KO) & 0.001 (Cx30 KO) \\
\hline
\end{tabular}

Normalized p-values by gene set enrichment analysis between WT (C57BL/6) and Cx30 KO mice are shown. Upregulated groups are indicated in parenthesis for each category.

\section{Microglia in Cx30 KO EAE Mice Have an Anti-inflammatory Phenotype in the Chronic Phase of EAE by Gene Expression Microarrays}

To further characterize the activated microglial phenotype in the chronic phase of EAE in $\mathrm{C} \times 30 \mathrm{KO}$ mice, we used $\mathrm{MOG}_{35}-55$-induced EAE and isolated microglia from the spinal cords and brains of Cx30 KO and WT mice at Day 39 after immunization, when clinical scores and AUC in the chronic phase were significantly lower in $\mathrm{Cx} 30 \mathrm{KO}$ mice than in WT mice $(p=0.0023)$. Microglia isolated from Cx30 KO EAE spinal cords and brains had lower expression levels of proinflammatory genes, such as $I L-1 b$, Nos2, Tnf, and Ptgs2, but no significant changes in anti-inflammatory gene levels, except for an increase in the Mrclgene (Figure 3F). GSEA analysis revealed that microglia from $\mathrm{Cx} 30 \mathrm{KO}$ mouse spinal cord and brain had less pro-inflammatory gene expressions than those from WT mice in the chronic EAE phase (spinal cord, ES $=0.721$, normalized $p=0.007, \mathrm{FDR}=0.018$; brain, proinflammatory genes; $\mathrm{ES}=0.643$, normalized $p=0.016$, FDR $=$ 0.037; Table 1 and Figures 3G-J). Furthermore, gene expressions of cytokines/chemokines, complements, alarmins, ROS, MHC, and tumor antigens in spinal cord but not brain microglia were significantly less in $\mathrm{Cx} 30 \mathrm{KO}$ than in WT mice in the chronic EAE phase (Table 1 and Supplementary Figures 3B,D). These findings indicate that the increased numbers of activated microglia in $\mathrm{C} \times 30 \mathrm{KO}$ mice have a reduced pro-inflammatory phenotype, especially in the spinal cord, in the chronic EAE phase.

\section{Cx30 KO Mice Upregulate Arginase-1 and BDNF but Downregulate NOS2 in the Chronic Phase of EAE}

To confirm the anti-inflammatory nature of the activated microglia in the CNS tissues of $\mathrm{Cx} 30 \mathrm{KO}$ mice in the chronic EAE phase, we performed double-staining for Iba-1 and arginase-1, an anti-inflammatory gene. Colocalization of Iba-1 and arginase- 1 was more frequently observed in the optic nerve and cerebellum of Cx30 KO mice compared with WT littermate mice $(p=0.012$, and $p=0.0041$, respectively; determined by higher mean Pearson's coefficient values; Figures 6A-D), although differences in colocalization levels between WT and $\mathrm{Cx} 30 \mathrm{KO}$ mice did not reach statistical significance in the spinal cord, possibly because of the infiltration of peripheral blood-borne macrophages (Figures 6E,F). Furthermore, in the optic nerve of $\mathrm{C} x 30 \mathrm{KO}$ mice, BDNF immunoreactivity was significantly greater compared with WT mice $(p=$ 0.0019; Supplementary Figures 5A,B). By contrast, NOS2 immunoreactivity in the optic nerve, cerebellum, and spinal cord white matter was significantly lower in $\mathrm{Cx} 30 \mathrm{KO}$ mice than in WT mice ( $p=0.0003, p=0.0032$, and $p=0.0047$, respectively; Supplementary Figures $\mathbf{5 C}-\mathbf{H}$ ). These findings indicate that microglia in Cx30 KO mice tended to have an anti-inflammatory phenotype, which is more evident in the CNS areas where peripheral blood-borne macrophages are rare during chronic EAE.

\section{Cx30 Deficiency Induces the Earlier and Stronger Activation of A2 Astrocytes During EAE}

Compared with naïve spinal cord, numbers of $\mathrm{GFAP}^{+}$astrocytes in the white and gray matter of the spinal cord increased significantly in the chronic EAE phase in both WT and Cx30 KO mice (white matter, $p=0.0159$ and $p=0.0003$, respectively; and gray matter, $p=0.0008$ and $p=0.0014$, respectively, at Day 59) but not in the acute phase, except for $\mathrm{GFAP}^{+}$astrocytes in the gray matter of Cx30 KO mice at Day 16 ( $p=0.0154$; Figure 7A; Supplementary Figures 6A-D). However, Cx30 KO mice had significantly more $\mathrm{GFAP}^{+}$astrocytes in the spinal cord white and gray matter (white matter; $p=0.0341$ on Day 13 and gray matter; $p=0.0006$ on Day 16) during the acute phase compared with WT mice, whereas this difference was not evident in the chronic phase in either the white or gray matter, suggesting the earlier and stronger activation of astrocytes in $\mathrm{Cx} 30 \mathrm{KO}$ mice (Figures 7A-C).

In WT mice, Cx30 immunoreactivity was unchanged throughout the clinical course of EAE in the spinal white matter, but compared with the naïve state it was increased significantly in the spinal cord gray matter in the chronic stage $(p=$ 0.0327; Figures 7A,D,E; Supplementary Figures 6E,F). In Cx30 KO mice, Cx30 immunoreactivity was not detected at any stage 
A

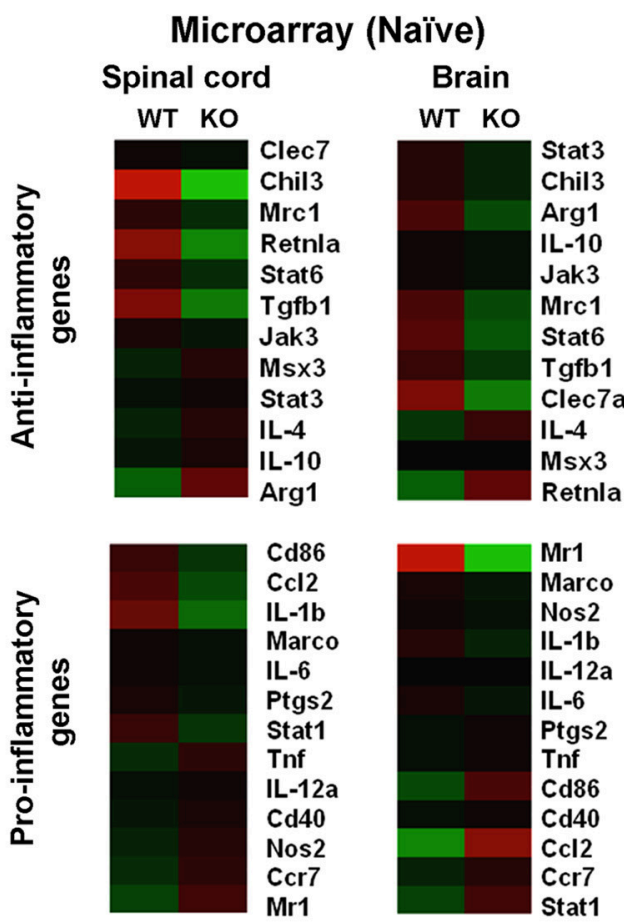

$\mathbf{F}$

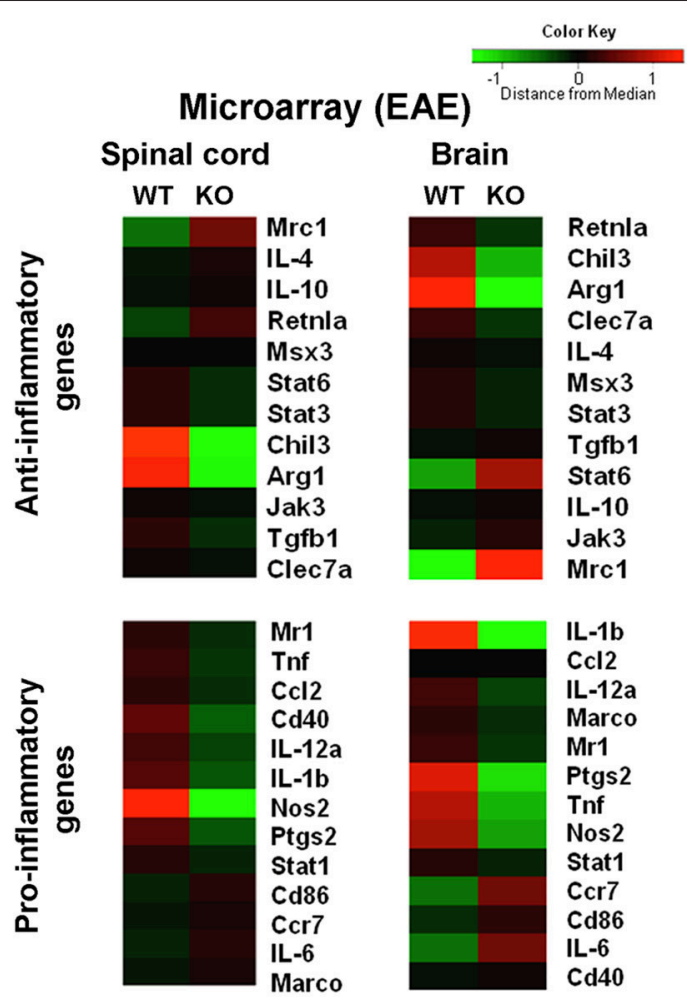

Enrichment plot: Anti-inflammatory (Spinal cord EAE)

H

Enrichment plot:

Anti-inflammatory (Brain EAE)

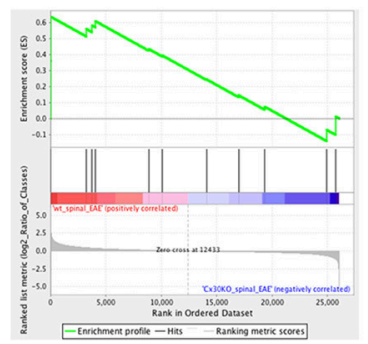

Enrichment plot:

Pro-inflammatory (Spinal cord EAE)

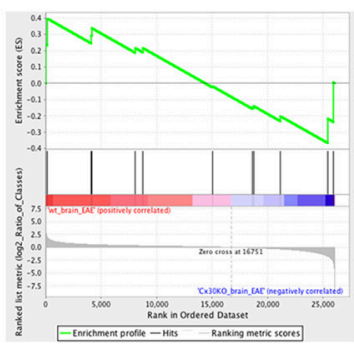

Enrichment plot:

Pro-inflammatory (Brain EAE)
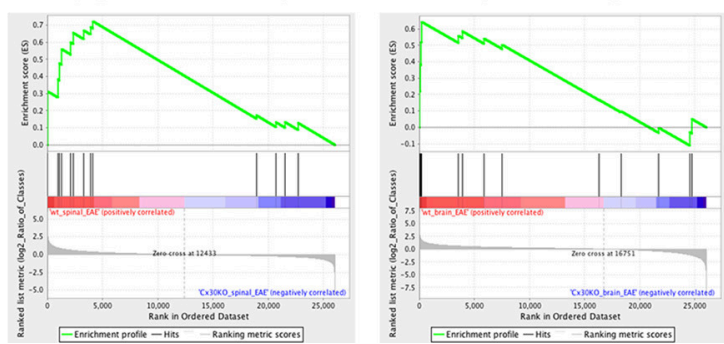

FIGURE 3 | Microarray analysis of microglia isolated from spinal cords and brains of WT (C57BL/6) and Cx30 KO mice. (A) Cluster analysis of gene expression arrays according to anti-inflammatory and pro-inflammatory genes of naive brain and spinal cord from WT (C57BL/6) and Cx30 KO mice. Color keys on each column represent $Z$ scores for each gene. (B-E) Enrichment plots for the anti-inflammatory (B,C) and pro-inflammatory (D,E) genes of naive spinal cords (B,D) and brains (C,E) from WT (C57BL/6) and Cx30 KO mice. (F) Cluster analysis of gene expression arrays according to anti-inflammatory and pro-inflammatory genes of chronic EAE brains and spinal cords from WT (C57BL/6) and CX30 KO mice. Color keys on each column represent $Z$ scores for each gene. (G-J) Enrichment plots for the anti-inflammatory $\mathbf{( G , H )}$ and pro-inflammatory $\mathbf{( I , J ) ~ g e n e s ~ o f ~ c h r o n i c ~ E A E ~ s p i n a l ~ c o r d s ~}(\mathbf{G}, \mathbf{I})$ and brains $\mathbf{( H , J )}$ from WT $(\mathbf{C} 57 \mathrm{BL} / 6)$ and $\mathrm{Cx30} \mathrm{KO}$ mice. The relative gene positions are indicated by the straight lines (line plot) under each graph. Lines clustered to the left represent higher ranked genes in the ranked list. 
A

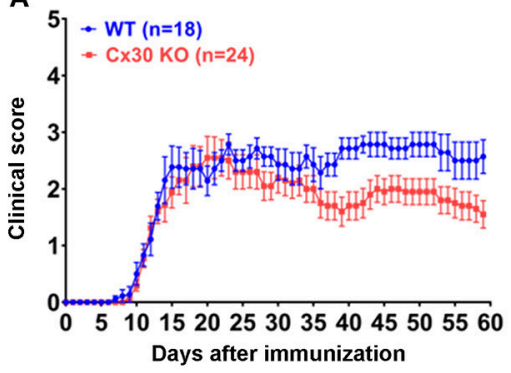

Days after immunization
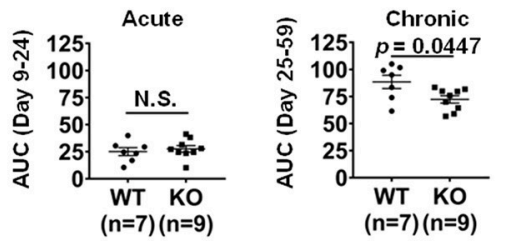

\begin{tabular}{llll}
\multicolumn{5}{c}{ Comparison of acute EAE between WT and Cx30 KO mice } \\
\hline Mice & Incidence (\%) & Day of onset & Peak clinical score \\
\hline WT & $18 / 18(100 \%)$ & $11.78 \pm 0.57$ & $2.86 \pm 0.18$ \\
Cx30 KO & $24 / 24(100 \%)$ & $11.71 \pm 0.44$ & $2.60 \pm 0.17$ \\
\hline
\end{tabular}
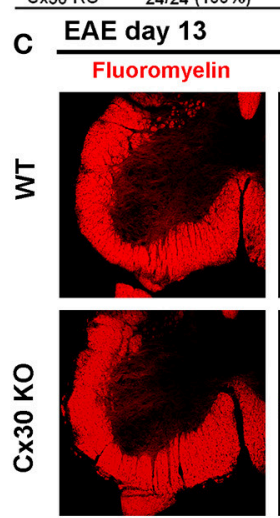

EAE day 59
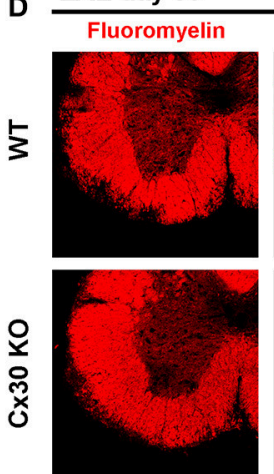

Fluoromyelin / CD 45 / DAPI

\section{B \\ EAE Day 59 H\&E}

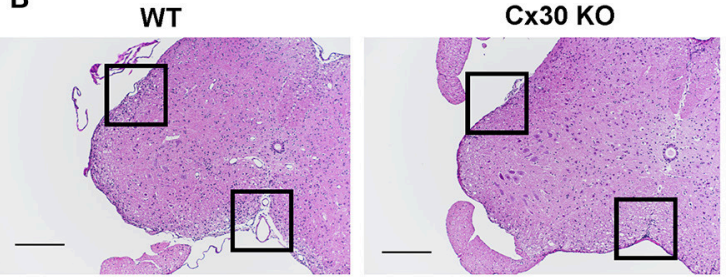

Higher magnification
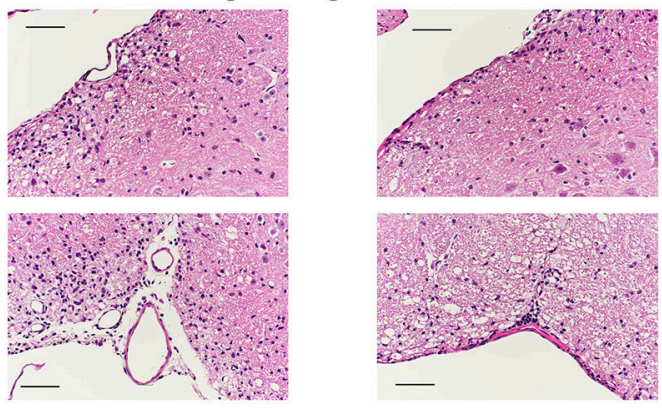

E

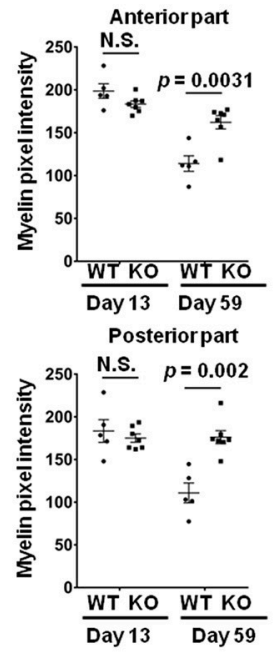

$\mathbf{F}$

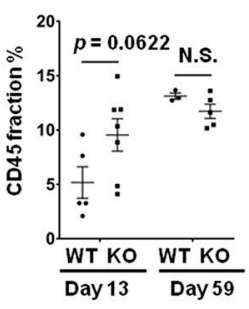

FIGURE 4 | Improvement of clinical severity and demyelination in the chronic but not acute phase of EAE in CX30 KO mice. (A) EAE clinical score changes in WT (littermate) and Cx30 KO mice. The severity of disease was separately analyzed according to acute (Days 9-24) and chronic (Days 25-59) phases by evaluating the area under the curve (AUC). Data shown are from a single experiment representative of four independent experiments including a total of 42 mice; $p$-values of the AUC were determined by the Mann-Whitney U-test. There was no significant difference in incidence, day of onset, or peak clinical score between WT (littermate) and CX30 KO mice in the acute EAE phase. Data for the following parameters are shown as the mean \pm S.E.M.: day of EAE onset, and peak clinical score of mice that developed EAE. Statistical differences were determined using the unpaired $t$-test with Welch's correction. (B) HE staining of spinal cords in the chronic EAE phase (Day 59). Scale bars, $200 \mu \mathrm{m}$ in the upper panels and $100 \mu \mathrm{m}$ in the lower panels. (C,D) Confocal images showing immunostaining for Fluoromyelin and CD45 in spinal cord sections from WT (littermate) and Cx30 KO mice in the acute (Day 13) and chronic (Day 59) EAE phases. Scale bars, $200 \mu \mathrm{m}$. (E) Quantification of myelin density in the anterior and posterior parts of spinal cords from WT (littermate) and Cx30 KO mice in the acute (Day 13) and chronic (Day 59) EAE phases. (F) Quantification of the CD45+ cell area fraction in the anterior spinal cords from WT (littermate) and Cx30 KO EAE mice in the acute (Day 13) and chronic EAE phases (Day 59). Statistical differences were determined using the unpaired $t$-test with Welch's correction. N.S. $=$ not significant. $n$ indicates the number of mice and each scatter dot represents individual mice in each group. 


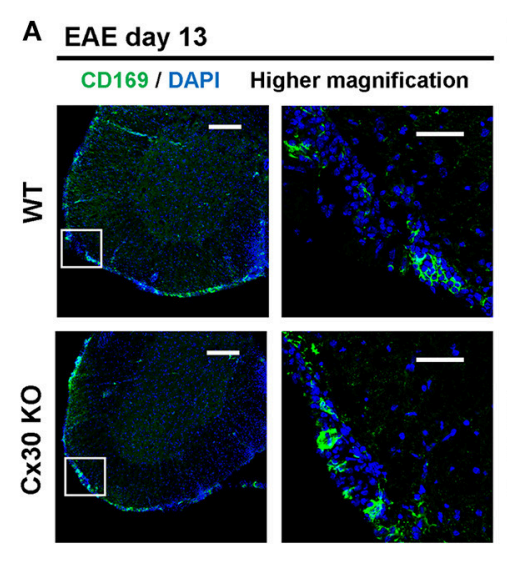

D EAE day 13

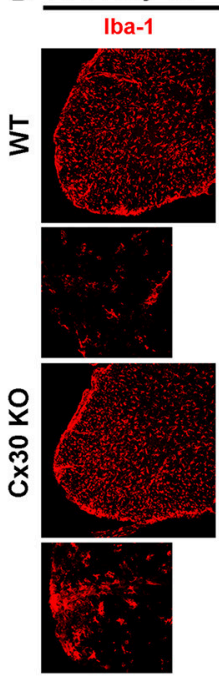

F
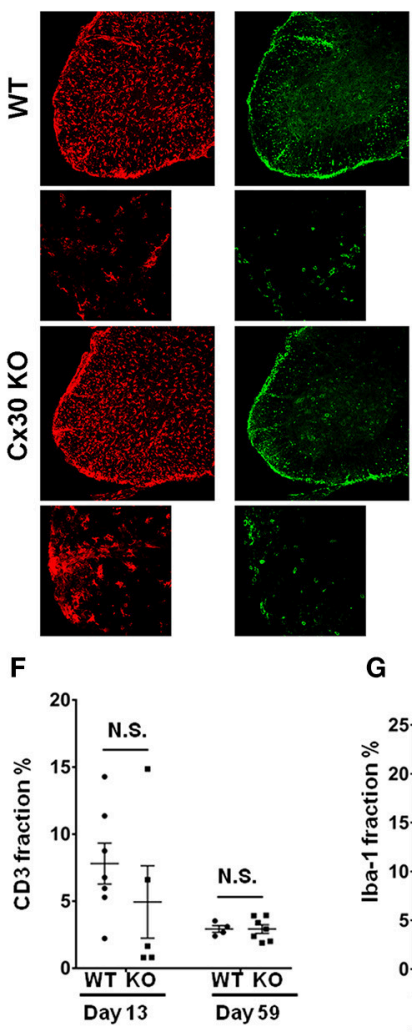

G
B EAE day 59
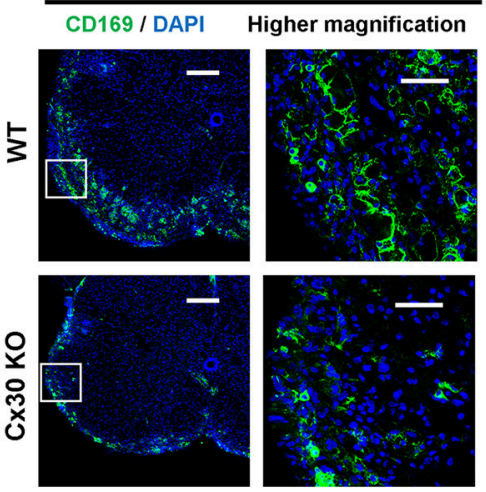

E EAE day 59

E EAE day
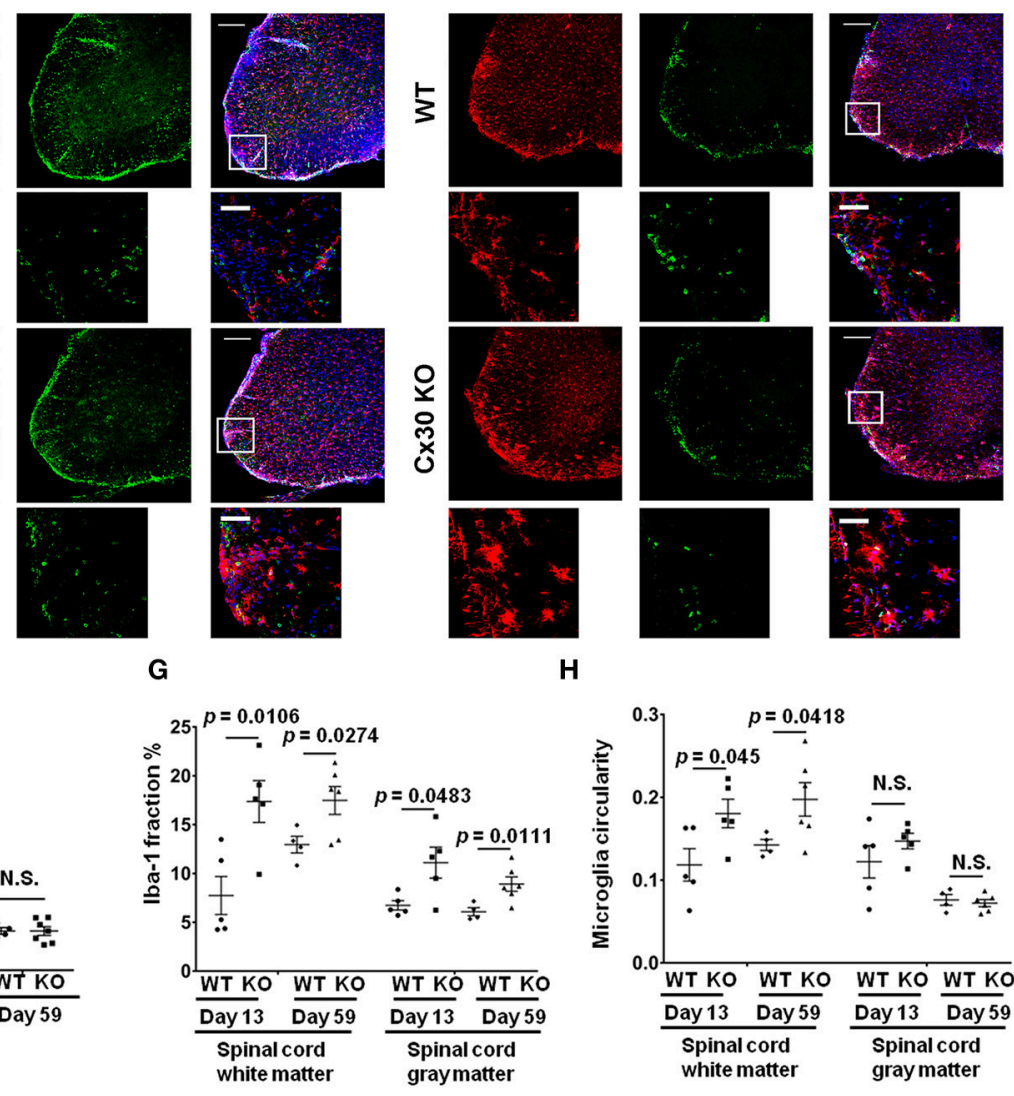
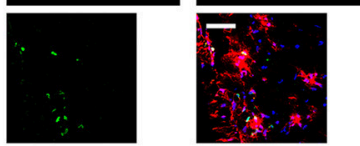

H

FIGURE 5 | Altered immune cell responses in CX30 KO mice in the chronic EAE phase compared with WT mice. (A,B) Confocal images showing immunostaining of CD169 in spinal cord sections from WT (littermate) and Cx30 KO EAE mice in the acute (Day 13) and chronic (Day 59) EAE phase. Scale bars, 200 $\mu$ m. Higher magnification images are CD169 in the spinal cord, which are highlighted by a white rectangular frame in the left panel of lower magnification images. (C) Quantification of the CD169+ cell area fraction in the anterior spinal cord white matter from WT (littermate) and Cx30 KO EAE mice in the acute (Day 13) and chronic (Day 59) EAE phases. (D,E) Confocal images showing immunostaining for lba-1 and CD3 in spinal cord sections from WT (littermate) and Cx30 KO EAE mice in the acute (Day 13) and chronic (Day 59) EAE phases. Scale bars, $200 \mu \mathrm{m}$. Higher magnification images show co-labeling of CD3 and Iba-1 in spinal cords, which are highlighted by a white rectangular frame in the lower magnification images above. (F) Quantification of the CD3 ${ }^{+}$cell area fraction in the anterior spinal cords from WT (littermate) and Cx30 KO EAE mice in the acute (Day 13) and chronic (Day 59) EAE phases. (G,H) Quantification of the lba-1+ cell area fraction (G) and microglial circularity $\mathbf{( H )}$ in the spinal cord white and gray matter from WT (littermate) and Cx30 KO EAE mice in the acute (Day 13) and chronic (Day 59) EAE phase. Means \pm S.E.M. are shown. Statistical differences were determined using the unpaired $t$-test with Welch's correction. N.S. $=$ not significant. $n$ indicates the number of mice and each scatter dot represents individual mice in each group. 

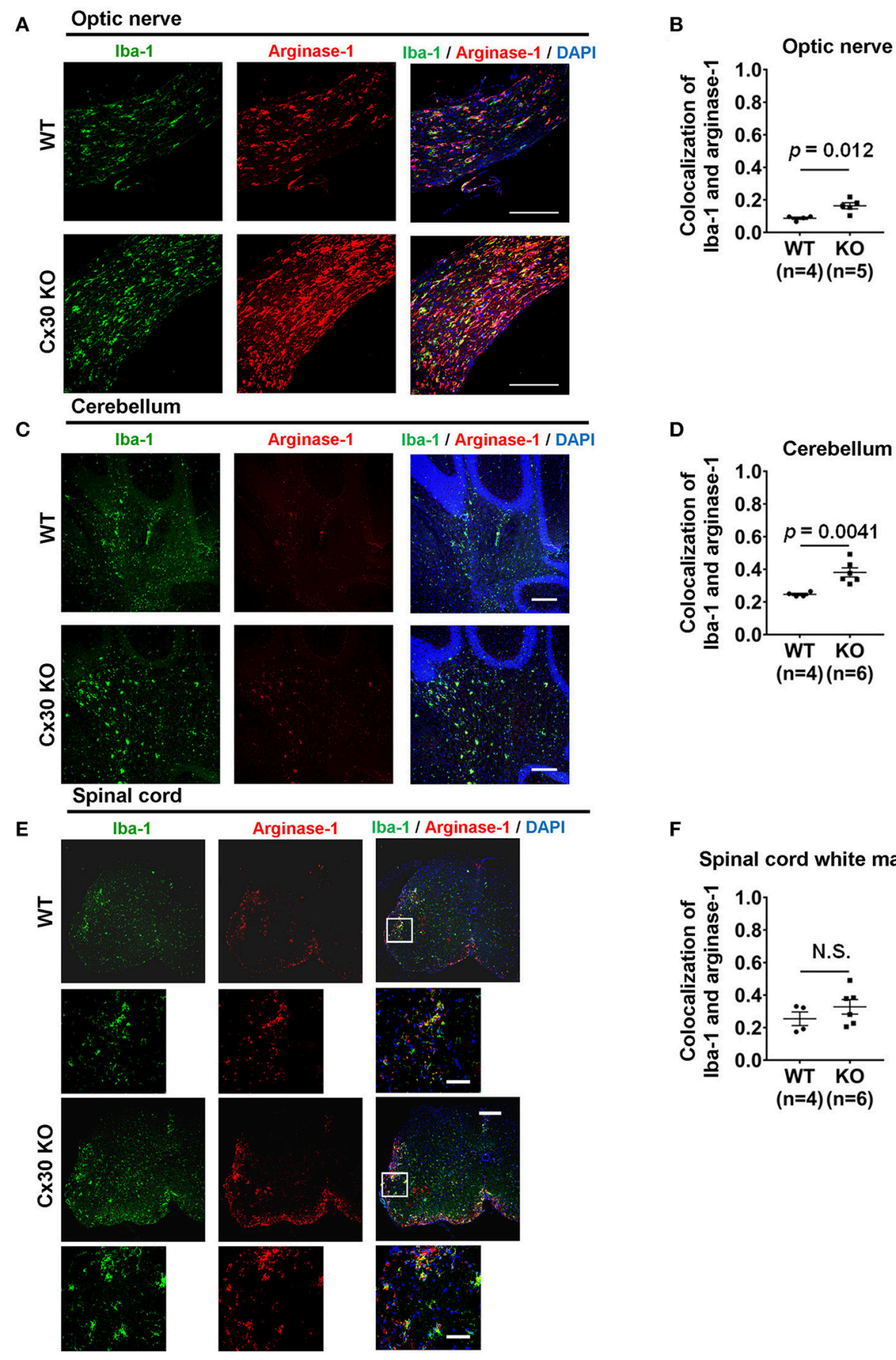

F

Spinal cord white matter

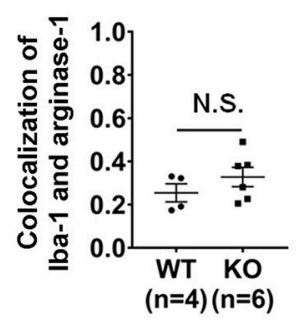

FIGURE 6 | Anti-inflammatory marker expression of microglia in WT and Cx30 KO mice with chronic EAE. (A,C,E) Confocal images showing immunostaining for Iba-1 and arginase-1 in optic nerves (A), cerebellum (C), and spinal cords (E) from WT (littermate) and Cx30 KO mice in the chronic EAE phase (Day 59). Scale bars, $200 \mu \mathrm{m}$. Each higher magnification image is from the white rectangular frame in the lower magnification image above. Scale bars in higher magnification images, 50 Mm. (B,D,F) Colocalization analysis of arginase-1 and lba-1 in optic nerves (B), cerebellum (D), and spinal cord white matter (F) from WT (littermate) and Cx30 KO mice in the chronic EAE phase, expressed as Pearson's correlation coefficients. Means \pm S.E.M. are shown. Statistical differences were determined using the unpaired $t$-test with Welch's correction. N.S. = not significant. $n$ indicates number of mice and each scatter dot represents individual mice in each group.

of EAE (Figures 7A,D,E). By contrast, Cx43 in the spinal cord white matter demonstrated a dynamic change during EAE in WT mice. $\mathrm{Cx} 43$ decreased significantly in the acute phase compared with the naïve state $(p=0.0149$ on Day 13 and $p=0.0067$ on Day 16) and then recovered to similar levels to the naïve state (significant increase compared with the peak day levels, 


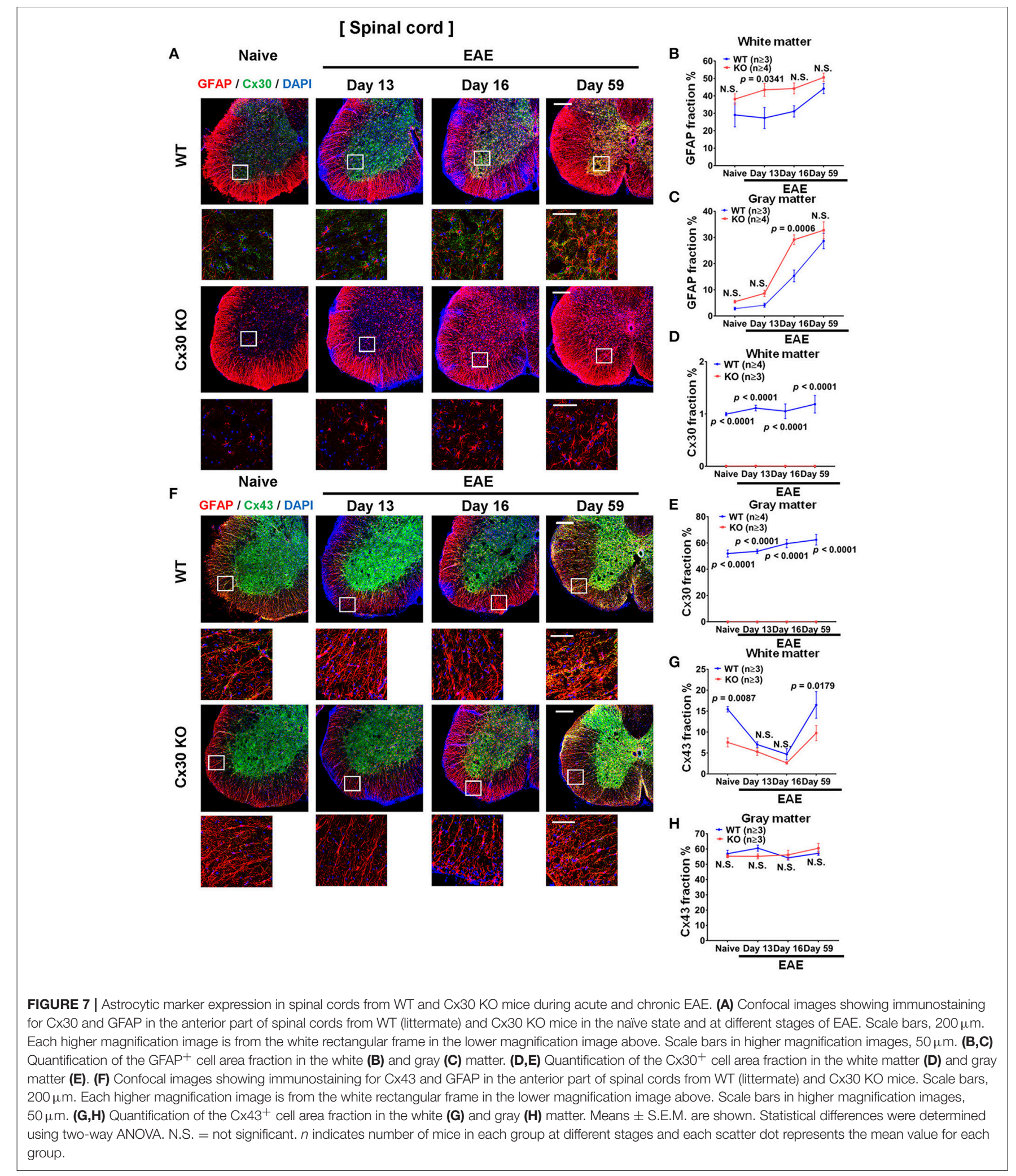

$p=0.0147$; Figures 7F,G; Supplementary Figure 6G). A similar decrease of $\mathrm{Cx} 43$ in the acute phase and recovery of $\mathrm{Cx} 43$ in the chronic phase were also observed in Cx30 KO mice ( $p=0.0142$ at Day 16 and $p=0.0026$ at Day 59 compared with Day 16; Figures 7F,G; Supplementary Figure 6I); however, Cx43 levels were significantly lower in Cx30 KO mice than in WT mice, in 
the naïve state and in the chronic EAE phase $(p=0.0087$ and $p=$ 0.0179 , respectively) (Figures 7F,G). Cx43 expression in the gray matter did not show obvious changes in either WT or Cx30 KO mice (Figures 7F,H; Supplementary Figures 6H,J).

We examined A1 and A2 astrocyte markers based on a recent report (53). Immunostaining analyses for S100A10, a representative $\mathrm{A} 2$ astrocyte marker, revealed a successive increase in the spinal cord white matter during the course of EAE, which was highest in the chronic EAE stage, in both WT and Cx30 KO mice compared with the naïve state (not significant in either mouse strain on Day 13; not significant and $p=0.0251$, respectively, on Day $16 ; p=$ 0.0010 and $p=0.0002$, respectively, on Day 59; Figures 8A,B; Supplementary Figures 6K,L). Interestingly, this increase in S100A10 immunoreactivity was greater in Cx30 KO mice than in WT mice at all stages of EAE and the difference increased in the later stages of $\operatorname{EAE}(p<0.0001$ at Day 59; Figures 8A,B). By contrast, $\mathrm{C} 3$, an A1 astrocyte marker, sharply increased and peaked in the acute phase and then steadily decreased in the chronic phase compared with the naïve state in both WT and Cx30 KO mice $(p=0.0174$ on Day 13 , and $p=0.0174$ on Day 16, respectively; Figures $\mathbf{8 C}, \mathbf{D}$; Supplementary Figures $6 \mathrm{M}, \mathrm{N}$ ). These findings suggest that the stronger activation of $\mathrm{A} 2$ astrocytes in $\mathrm{Cx} 30 \mathrm{KO}$ mice plays a role in inducing neuroprotective microglia, which attenuate EAE in the chronic phase.

\section{Cx30 Deficiency Causes Less Neuronal Death in the Chronic Phase of EAE}

Finally, because astrocytic Cx30 has close contact with neurons, we examined changes in neurons and axons in $\mathrm{Cx} 30 \mathrm{KO}$ mice during EAE. In the native state, the numbers of $\mathrm{NeuN}^{+}$cells and SMI- $31^{+}$axonal density were not significantly different between Cx30 and WT littermate mice (Figures 9A-D). Although only SMI- $31^{+}$axonal density decreased significantly in the chronic EAE phase compared with the naïve state in both WT and Cx30 KO mice ( $p<0.0001$, and $p=0.0035$, respectively), Cx30 KO mice had significantly more $\mathrm{NeuN}^{+}$cells and SMI- $31^{+}$remaining axons compared with WT mice in the chronic EAE phase $(p=0.0376$, and $p=0.0003$, respectively; Figures 9E,F). Because IL-34, expressed on CNS neurons, induces the differentiation of microglia to a neuroprotective phenotype (54), we examined its expression by immunostaining, and detected significantly more IL-34 in the spinal white matter of $\mathrm{Cx} 30 \mathrm{KO}$ mice than in WT mice in the chronic EAE phase (Supplementary Figure 7). These findings suggest less neuronal death and axonal loss in Cx30 KO mice compared with WT mice in the chronic phase of EAE, leading to more IL-34 production in Cx30 KO mice.

\section{DISCUSSION}

The main new findings in the present study are as follows: (1) Cx30 deficiency attenuated only chronic EAE clinically and pathologically without affecting $\mathrm{T}$ cell infiltration. (2) Cx30 deficiency increased the numbers of ramified microglia in the naïve state and induced earlier and more widespread activation of microglia in the acute and chronic phases of EAE. (3) These activated microglia in $\mathrm{Cx} 30 \mathrm{KO}$ mice were prone to differentiate toward an anti-inflammatory phenotype with less pro-inflammatory gene expression. (4) In the naïve state, Cx30 deficiency induced only a modest enlargement of astrocytic processes in the spinal gray matter and a partial reduction of $\mathrm{Cx} 43$ expression in the spinal white matter, whereas it caused earlier and stronger activation of astrocytes in the acute EAE phase, upregulated S100A10, a representative $\mathrm{A} 2$ astrocyte marker, and attenuated the recovery of $\mathrm{Cx} 43$ in the chronic phase. (5) Cx30 deficiency rescued more neurons and axons in the chronic EAE phase without influencing the quantity of neurons or axons in the naïve state.

According to the present study, Cx30 deficiency has no apparent influence on the clinical and histological severity of acute EAE, in accordance with a previous report describing that a single or double $\mathrm{KO}$ of $\mathrm{Cx} 30$ and/or $\mathrm{Cx} 43$ did not affect acute EAE (29). Collectively, this suggests that Cx30 does not modulate the peripheral immune system or alter the clinical course of acute EAE. Surprisingly, chronic EAE and demyelination were significantly attenuated by $\mathrm{Cx} 30$ deficiency. These differences in results between a previous study (29) and the current study might be attributable to a difference in the observation period. Thus, acute and chronic EAE are differentially regulated, at least in part, and Cx30 is mainly involved in the chronic phase, when microglia and astrocytes are postulated to be key players $(55,56)$.

We demonstrated the attenuation of chronic EAE by $\mathrm{Cx} 30$ deficiency without influencing $\mathrm{T}$ cell infiltration, which further underlines the importance of microglia and astrocytes in the chronic phase of EAE. In naïve $\mathrm{Cx} 30 \mathrm{KO}$ mice, increased microglia were observed throughout the CNS; however, these microglia retained a ramified morphology without a significant increase in circularity. Consistent with this morphology, they had similar expression levels of pro-inflammatory genes to WT microglia by RNA microarray. During the chronic EAE phase in $\mathrm{Cx} 30 \mathrm{KO}$ mice, there were increased numbers and more activated microglia in the spinal cord and the brain, which had reduced pro-inflammatory gene expression compared with WT mice. Indeed, microglia in Cx30 KO mice had lower expressions of $I L$ 1b, Nos2, Tnf, and Ptgs 2 and a higher expression of Mrc1. IL-1 $\beta$, tumor necrosis factor- $\alpha$, NOS2, and prostaglandin-endoperoxide synthase-2, also known as cyclooxygenase-2, are well-known proinflammatory molecules involved in neuroinflammation (5761). Mannose receptor C-type 1 (Mrc1), downregulated by IFN- $\gamma$ (62), and upregulated by IL-4 (63), is expressed at high levels during the resolution of inflammation where it has a critical role in the removal of inflammatory glycoproteins (64). We also immunohistochemically observed the increased expression of arginase- 1 and BDNF and the decreased expression of NOS2 in the optic nerve, cerebrum, and cerebellum. Although such changes were not clear in inflamed spinal cord lesions, the increased infiltration of peripheral blood-borne proinflammatory macrophages, reported to be abundant in chronic EAE (65) and shown as a significant increase of CD169 ${ }^{+}$cells in the chronic EAE phase in the present study, might have obscured 


\section{[ Spinal cord]}

A
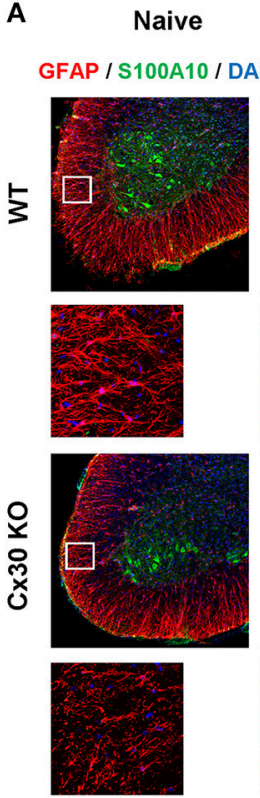

C
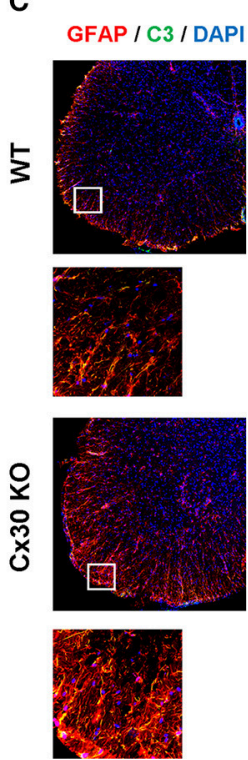

EAE
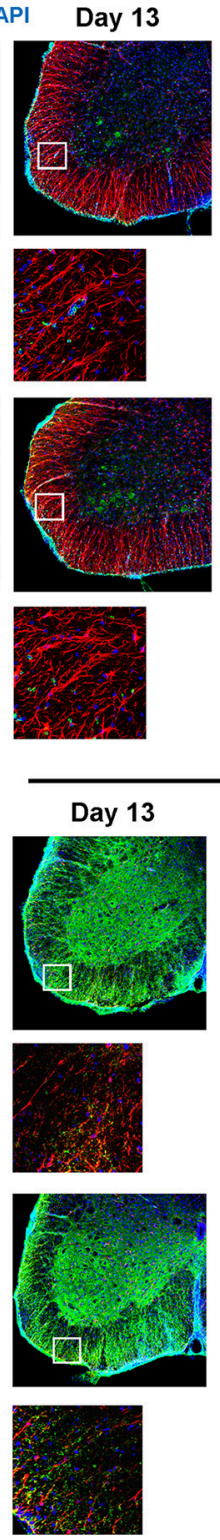

Day 16
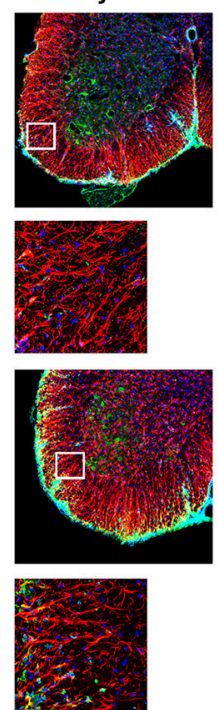

EAE
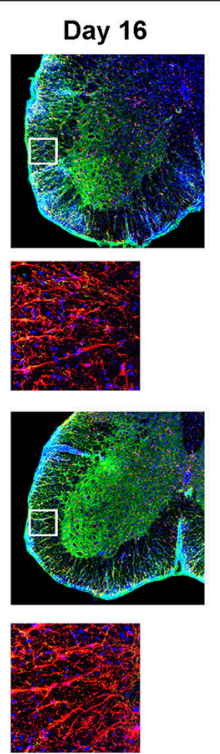
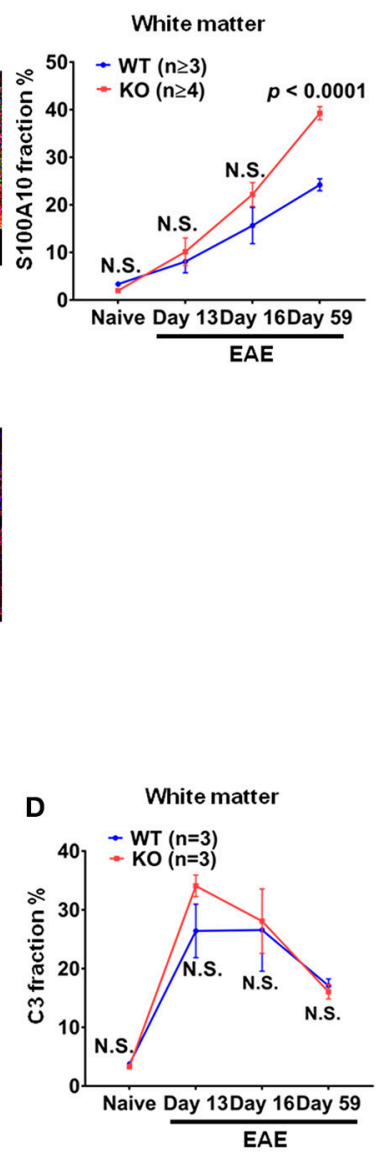

FIGURE 8 | A1 and A2 astrocyte marker expression in spinal cords from WT and Cx30 KO mice during acute and chronic EAE. (A) Confocal images showing immunostaining for S100A10 and GFAP in spinal cords from WT (littermate) and Cx30 KO mice. Scale bars, $200 \mu \mathrm{m}$. Each higher magnification image is from the white rectangular frame in the lower magnification image above. Scale bars in higher magnification images, $50 \mu \mathrm{m}$. (B) Quantification of the S100A10 ${ }^{+}$cell fraction in the spinal cord white matter. (C) Confocal images showing immunostaining for GFAP and C3 in spinal cords from WT (littermate) and Cx30 KO mice. Scale bars, $200 \mu \mathrm{m}$. (D) Quantification of the $\mathrm{C}^{+}$cell fraction in the spinal cord white matter. Each higher magnification image is from the white rectangular frame in the lower magnification image above. Scale bars in higher magnification images, $50 \mu \mathrm{m}$. Means \pm S.E.M. are shown. Statistical differences were determined using two-way ANOVA. N.S. $=$ not significant. $n$ indicates number of mice in each group at different stages and each scatter dot represents the mean value for each group.

the reduced pro-inflammatory nature of $\mathrm{Cx} 30 \mathrm{KO}$ mouse spinal cord microglia.

Notably, spinal cord but not brain microglia had significantly lower expressions of cytokines/chemokines, alarmins, and MHC genes in $\mathrm{Cx} 30 \mathrm{KO}$ than in WT mice, suggesting spinal cord microglia are less reactive to inflammatory insults than brain microglia in the naïve state in $\mathrm{Cx} 30 \mathrm{KO}$ mice. In the chronic EAE phase, cytokines/chemokines, complements, alarmins, ROS, and $\mathrm{MHC}$ gene expressions in microglia were significantly lower in $\mathrm{Cx} 30 \mathrm{KO}$ than in WT mice in the spinal cord but not in the brain, 


\section{A Naive}
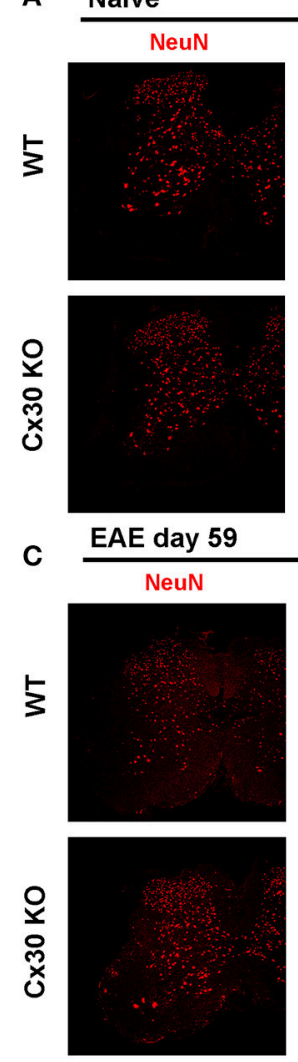

Cx30
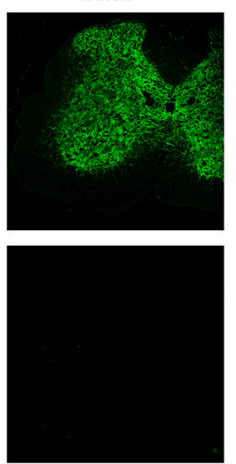

$\mathrm{C} \times 30$
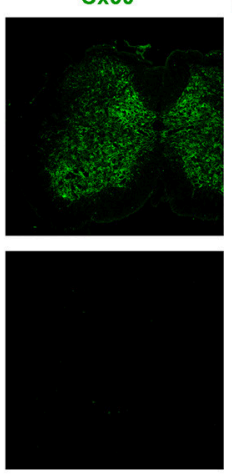

NeuN / C $\times 30 /$ DAPI
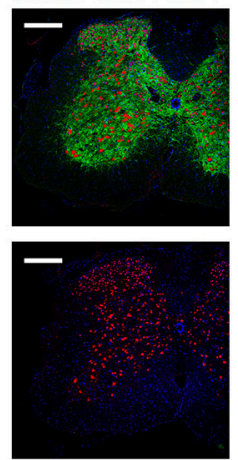

NeuN / C X30 / DAPI
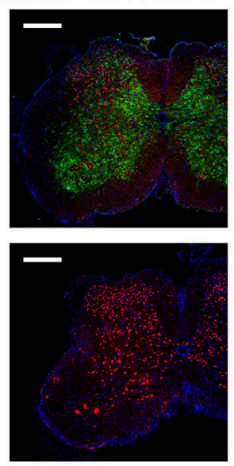

B Naive

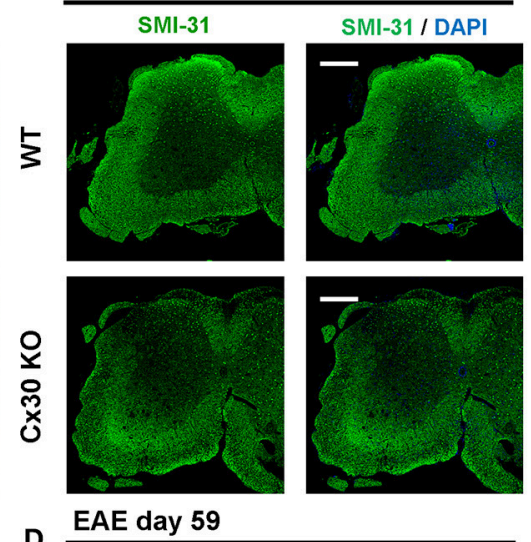

D SMI-31
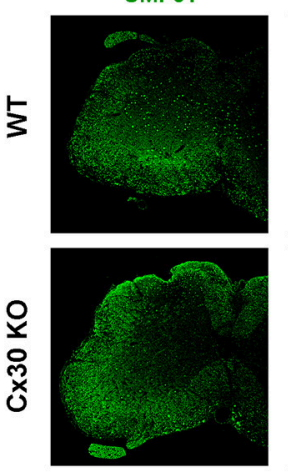
SMI-31/ DAPI

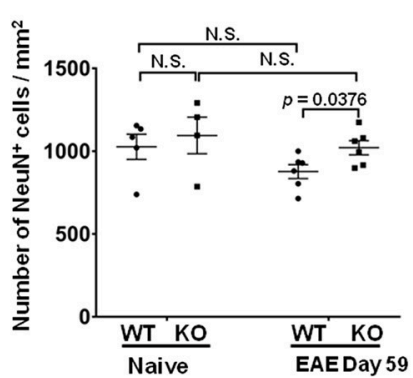

$\mathbf{F}$

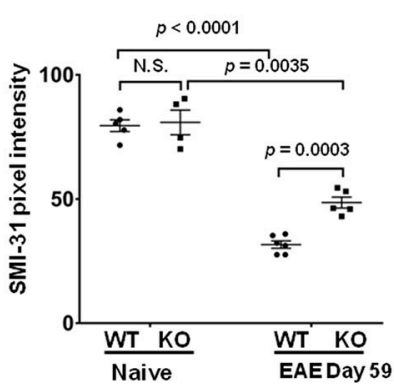

FIGURE 9 | NeuN and SMI-31 immunostaining of spinal cords from WT and Cx30 KO mice with or without EAE. (A,C) Confocal images showing immunostaining for Cx30 and NeuN in spinal cords from WT (littermate) and Cx30 KO mice in the naive state (A) and chronic EAE phase (Day 59) (C). Scale bars, $200 \mu \mathrm{m}$.

(B,D) Confocal images showing immunostaining for SMI-31 in spinal cord sections from WT (littermate) and Cx30 KO EAE mice in the naive state (B) and chronic EAE phase (Day 59) (D). Scale bars, $200 \mu \mathrm{m}$. (E) Quantification of NeuN ${ }^{+}$cell numbers $/ \mathrm{mm}^{2}$ of spinal cord in the naive state or chronic EAE phase. (F) Quantification of SMI-31 density in anterior white matter regions of the spinal cord from WT (littermate) and CX30 KO EAE mice in the naive state and chronic EAE phase (Day 59). Means \pm S.E.M. are shown. Statistical differences were determined using the unpaired $t$-test with Welch's correction. N.S. $=$ not significant. $n$ indicates the number of mice and each scatter dot represents individual mice in each group.

indicating that spinal cord microglia have a tendency to have a reduced pro-inflammatory and increased anti-inflammatory phenotype compared with brain microglia in Cx30 KO mice.

Earlier and stronger activation of astrocytes in EAE was another characteristic feature of Cx30 KO mice. S100A10, a neuroprotective A2 astrocyte-related gene product (53), was increasingly expressed in the spinal cord white matter as EAE proceeded from the acute to chronic phase. Although the precise functions of astrocytic S100A10 remain to be elucidated, it was reported to be required for membrane repair (66), cell proliferation (67), and inhibition of cell apoptosis by interaction with $\mathrm{Bcl}-\mathrm{xL} / \mathrm{Bcl}$-2-associated death promoter (68). Thus, S100A10 expressed in astrocytes might be beneficial for tissue repair. These findings collectively suggest that $\mathrm{Cx} 30$-deficient astrocytes may be prone to differentiate toward an A2 astrocyte phenotype upon activation. In addition, these $\mathrm{Cx} 30$-deficient astrocytes showed less $\mathrm{Cx} 43$ immunoreactivity in the astrogliotic scar in the chronic 
EAE phase as well as in the naïve state. The overexpression of $\mathrm{Cx} 43$ might propagate inflammatory mediators through homotypic Cx43 GJ channels and Cx43 hemichannels (69-72). Thus, the downregulation of Cx43 on Cx30deficient astrocytes may also function in suppressing chronic neuroinflammation.

Increased numbers of $\mathrm{GFAP}^{+}$astrocytes were present in the white matter compared with the gray matter of $\mathrm{Cx} 30$ KO mice spinal cords. Furthermore, increased S100A10 and decreased Cx43 levels were observed in the spinal white matter, suggesting white matter astrocytes may exhibit earlier and stronger activation toward an A2 phenotype than gray matter astrocytes in the $\mathrm{Cx} 30 \mathrm{KO}$ spinal cord. Because increased microglia numbers were observed in spinal white and gray matter in $\mathrm{Cx} 30 \mathrm{KO}$ mice while a circularity increase was detected only in white matter microglia, the activation of microglia toward an anti-inflammatory phenotype may occur more strongly in the spinal white matter than in the gray matter. Collectively, earlier and stronger astroglial activation toward an A2 phenotype occurred in the spinal white matter, which might partly explain the induction of anti-inflammatory microglia in Cx30 KO mice spinal white matter. Such microglial activation occurred even in the acute EAE phase in Cx30 KO mice; however, this effect might not be sufficient in the acute phase when $\mathrm{CD}^{+} \mathrm{T}$ cells mainly induce acute inflammation, while becoming evident in the chronic phase when glial inflammation predominates.

Interestingly, Cx30 KO mice had more remaining neurons and axons than WT mice in the chronic phase of EAE. Cx30 on astrocytes exists in close proximity to neurons (32) and Cx30 hemichannels release glutamate that exerts excitotoxicity on neurons $(54,73)$. Thus, Cx30 deficiency may confer survival of neurons and axons. Neuronal IL-34 is a potent activator for microglia toward a neuroprotective phenotype (74). Accordingly, we hypothesized that the earlier and stronger activation of astroglia toward an $\mathrm{A} 2$ phenotype in $\mathrm{Cx} 30 \mathrm{KO}$ mice in the spinal white matter induced anti-inflammatory microglia $(75,76)$. Increased axonal IL-34 in Cx30 KO mice may also contribute to

\section{REFERENCES}

1. Compston A, Coles A. Multiple sclerosis. Lancet (2008) 372:1502-17. doi: 10.1016/s0140-6736(08)61620-7

2. Tremlett H, Zhao Y, Devonshire V. Natural history of secondaryprogressive multiple sclerosis. Multiple Sclerosis J. (2008) 14:314-24. doi: $10.1177 / 1352458507084264$

3. Scalfari A, Neuhaus A, Daumer M, Muraro PA, Ebers GC. Onset of secondary progressive phase and long-term evolution of multiple sclerosis. J Neurol Neurosurg Psychiatr. (2013) 85:67-75. doi: 10.1136/jnnp-2012-304333

4. Kappos L, Kuhle J, Multanen J, Kremenchutzky M, di Cantogno E, Cornelisse V. et al. Factors influencing long-term outcomes in relapsingremitting multiple sclerosis: PRISMS-15. J Neurol Neurosurg Psychiatr. (2015) 86:1202-7. doi: 10.1136/jnnp-2014-310024

5. Lorscheider J, Jokubaitis VG, Spelman T, Izquierdo G, Lugaresi A, Havrdova E, et al. Anti-inflammatory disease-modifying treatment and short-term disability progression in SPMS. Neurology (2017) 89:1050-9. doi: 10.1212/WNL.0000000000004330 the induction of anti-inflammatory microglia in the chronic EAE phase (74), which might dampen chronic neuroinflammation.

In conclusion, $\mathrm{Cx} 30$ on astrocytes plays a significant role in perpetuating neuroinflammation in chronic EAE by augmenting pro-inflammatory glial responses. How Cx30 deficiency attenuates inflammatory glial responses should be investigated in future studies, and might provide valuable information for new therapeutic strategies for chronic glial inflammation in MS.

\section{AUTHOR CONTRIBUTIONS}

MF, RY, GL, KM, HY, and AF performed experiments. MF, RY, HY, and JK designed the research. MF, RY, KM, NI, and JK analyzed the data and provided scientific suggestions. MF, $\mathrm{RY}$, and JK drafted the manuscript. All authors reviewed the manuscript.

\section{ACKNOWLEDGMENTS}

This work was supported in part by Glial Assembly Grantsin-Aid for Scientific Research on Innovative Areas (MEXT KAKENHI Grant Numbers 25117001 and 25117012) from the Ministry of Education, Culture, Sports, Science and Technology of Japan, and a Grant-in-Aid for Scientific Research (A) (JSPS KAKENHI Grant Number 16H02657) from the Japan Society for the Promotion of Science. We appreciate the technical assistance of The Research Support Center, Research Center for Human Disease Modeling, Kyushu University Graduate School of Medical Sciences. We thank Ann Turnley, PhD, from Edanz Group (www.edanzediting.com/ac) for editing a draft of this manuscript.

\section{SUPPLEMENTARY MATERIAL}

The Supplementary Material for this article can be found online at: https://www.frontiersin.org/articles/10.3389/fimmu. 2018.02588/full\#supplementary-material
6. Ontaneda D, Fox RJ, Chataway J. Clinical trials in progressive multiple sclerosis: lessons learned and future perspectives. Lancet Neurol. (2015) 14:208-223. doi: 10.1016/S1474-4422(14)70264-9

7. Kappos L, Bar-Or A, Cree BA, Fox RJ, Giovannoni G, Gold R, et al. Siponimod versus placebo in secondary progressive multiple sclerosis (EXPAND): a double-blind, randomised, phase 3 study. Lancet (2018) 391:1263-73. doi: 10.1016/S0140-6736(18)30475-6

8. Cohen JA, Arnold DL, Comi G, Bar-Or A, Gujrathi S, Hartung JP, et al. Safety and efficacy of the selective sphingosine 1-phosphate receptor modulator ozanimod in relapsing multiple sclerosis (RADIANCE): a randomised, placebo-controlled, phase 2 trial. Lancet Neurol. (2016) 15:373-81. doi: 10.1016/S1474-4422(16)00018-1

9. O'sullivan SA, O'sullivan C, Healy LM, Dev KK, Sheridan GK. Sphingosine 1phosphate receptors regulate TLR 4-induced CXCL 5 release from astrocytes and microglia. J Neurochem. (2018) 144:736-47. doi: 10.1111/jnc.14313

10. Healy LM, Sheridan GK, Pritchard AJ, Rutkowska A, Mullershausen F, Dev KK. Pathway specific modulation of S1P1 receptor signalling in rat and human astrocytes. Br J Pharmacol. (2013) 169:1114-29. doi: 10.1111/bph.12207 
11. Choi JW, Gardell SE, Herr DR, Rivera R, Lee C-W, Noguchi K, et al. FTY720 (fingolimod) efficacy in an animal model of multiple sclerosis requires astrocyte sphingosine 1-phosphate receptor 1 (S1P1) modulation. Proc Natl Acad Sci. (2011) 108:751-6. doi: 10.1073/pnas.1014154108

12. Mahad DH, Trapp BD, Lassmann H. Pathological mechanisms in progressive multiple sclerosis. Lancet Neurol. (2015) 14:183-93. doi: 10.1016/S1474-4422(14)70256-X

13. Prineas JW, Kwon EE, Cho ES, Sharer LR, Barnett MH, Oleszak EL, et al. Immunopathology of secondary-progressive multiple sclerosis. Ann Neurol. (2001) 50:646-57. doi: 10.1002/ana.1255

14. J.-Hervé C, Derangeon M. Gap-junction-mediated cell-to-cell communication. Cell Tissue Res. (2013) 352:21-31. doi: 10.1007/s00441012-1485-6

15. Nagy J, Patel D, Ochalski P, Stelmack G. Connexin 30 in rodent, cat and human brain: selective expression in gray matter astrocytes, co-localization with connexin43 at gap junctions and late developmental appearance. Neuroscience (1999) 88:447-68. doi: 10.1016/S0306-4522(98)00191-2

16. Rash J, Yasumura T, Davidson K, Furman C, Dudek FE, Nagy J. Identification of cells expressing Cx43, Cx30, Cx26, Cx32 and Cx36 in gap junctions of rat brain and spinal cord. Cell Commun Adhesion (2001) 8:315-20. doi: 10.3109/15419060109080745

17. Rouach N, Avignone E, Meme W, Koulakoff A, Venance L, Blomstrand $\mathrm{F}$, et al. Gap junctions and connexin expression in the normal and pathological central nervous system. Biol Cell (2002) 94:457-75. doi: 10.1016/S0248-4900(02)00016-3

18. Cotrina ML, Lin JH-C, Alves-Rodrigues A, Liu S, Li J, Azmi-Ghadimi H, et al. Connexins regulate calcium signaling by controlling ATP release. Proc Natl Acad Sci. (1998) 95:15735-40. doi: 10.1073/pnas.95.26.15735

19. Spray D, Harris A, Bennett M. Gap junctional conductance is a simple and sensitive function of intracellular pScience (1981) 211:712-5. doi: $10.1126 /$ science. 6779379

20. Stout CE, Costantin JL, Naus CC, Charles AC. Intercellular calcium signaling in astrocytes via ATP release through connexin hemichannels. J Biol Chem. (2002) 277:10482-8. doi: 10.1074/jbc.M109902200

21. Markoullis K, Sargiannidou I, Schiza N, Hadjisavvas A, Roncaroli F, Reynolds R, et al. Gap junction pathology in multiple sclerosis lesions and normal-appearing white matter. Acta Neuropathol. (2012) 123:873-86. doi: 10.1007/s00401-012-0978-4

22. Masaki K, Suzuki SO, Matsushita T, Matsuoka T, Imamura S, Yamasaki $\mathrm{R}$, et al. Connexin 43 astrocytopathy linked to rapidly progressive multiple sclerosis and neuromyelitis optica. PLoS ONE (2013) 8:e72919. doi: 10.1371/journal.pone.0072919

23. Masaki K, Suzuki SO, Matsushita T, Yonekawa T, Matsuoka T, Isobe $\mathrm{N}$, et al. Extensive loss of connexins in Balós disease: evidence for an auto-antibody-independent astrocytopathy via impaired astrocyteoligodendrocyte/myelin interaction. Acta Neuropathol. (2012) 123:887-900. doi: 10.1007/s00401-012-0972-x

24. Brand-Schieber E, Werner P, Iacobas DA, Iacobas S, Beelitz M, Lowery SL, et al. Connexin43, the major gap junction protein of astrocytes, is downregulated in inflamed white matter in an animal model of multiple sclerosis. $J$ Neurosci Res. (2005) 80:798-808. doi: 10.1002/jnr.20474

25. Markoullis K, Sargiannidou I, Gardner C, Hadjisavvas A, Reynolds R, Kleopa KA. Disruption of oligodendrocyte gap junctions in experimental autoimmune encephalomyelitis. Glia (2012) 60:1053-66. doi: 10.1002/glia.22334

26. Roscoe W, Kidder G, Karlik S. Experimental allergic encephalomyelitis in connexin 43-heterozygous mice. Cell Commun Adhesion (2007) 14:57-73. doi: 10.1080/15419060701459569

27. Roscoe W, Messersmith E, Meyer-Franke A, Wipke B, Karlik S. Connexin 43 gap junction proteins are up-regulated in remyelinating spinal cord. J Neurosci Res. (2007) 85:945-953. doi: 10.1002/jnr.21194

28. Theotokis P, Kleopa KA, Touloumi O, Lagoudaki R, Lourbopoulos A, Nousiopoulou E, et al. Connexin43 and connexin47 alterations after neural precursor cells transplantation in experimental autoimmune encephalomyelitis. Glia (2015) 63:1772-83. doi: 10.1002/glia.22843

29. Lutz SE, Raine CS, Brosnan CF. Loss of astrocyte connexins 43 and 30 does not significantly alter susceptibility or severity of acute experimental autoimmune encephalomyelitis in mice. J Neuroimmunol. (2012) 245:8-14. doi: 10.1016/j.jneuroim.2012.01.007

30. Dahl E, Manthey D, Chen Y, Schwarz H-J, Chang YS, Lalley PA, et al. Molecular cloning and functional expression of mouse connexin-30, a gap junction gene highly expressed in adult brain and skin. J Biol Chem. (1996) 271:17903-10. doi: 10.1074/jbc.271.30.17903

31. Dermietzel R, Traub O, Hwang T, Beyer E, Bennett M, Spray D, et al. Differential expression of three gap junction proteins in developing and mature brain tissues. Proc Natl Acad Sci. (1989) 86:10148-52. doi: $10.1073 /$ pnas.86.24.10148

32. Koulakoff A, Ezan P, Giaume C. Neurons control the expression of connexin 30 and connexin 43 in mouse cortical astrocytes. Glia (2008) 56:1299-311. doi: 10.1002/glia.20698

33. Kunzelmann P, Schröder W, Traub O, Steinhäuser C, Dermietzel R, Willecke $\mathrm{K}$. Late onset and increasing expression of the gap junction protein connexin30 in adult murine brain and long-term cultured astrocytes. Glia (1999) 25:111-9.

34. Watanabe M, Masaki K, Yamasaki R, Kawanokuchi J, Takeuchi H, Matsushita T, et al. Th1 cells downregulate connexin 43 gap junctions in astrocytes via microglial activation. Sci Rep. (2016) 6:38387. doi: 10.1038/ srep38387

35. Pannasch U, Freche D, Dallérac G, Ghézali G, Escartin C, Ezan P, et al. Connexin 30 sets synaptic strength by controlling astroglial synapse invasion. Nat Neurosci. (2014) 17:549-58. doi: 10.1038/nn.3662

36. Kardami E, Dang $\mathrm{X}$, Iacobas DA, Nickel BE, Jeyaraman $M$, Srisakuldee $\mathrm{W}$, et al. The role of connexins in controlling cell growth and gene expression. Prog Biophys Mol Biol. (2007) 94:245-64. doi: 10.1016/j.pbiomolbio.2007.03.009

37. Zhou JZ, Jiang JX. Gap junction and hemichannel-independent actions of connexins on cell and tissue functions-an update. FEBS Lett. (2014) 588:118692. doi: 10.1016/j.febslet.2014.01.001

38. Teubner B, Michel V, Pesch J, Lautermann J, Cohen-Salmon M, Söhl $\mathrm{G}$, et al. Connexin30 (Gjb6)-deficiency causes severe hearing impairment and lack of endocochlear potential. Hum Mol Genet. (2003) 12:13-21. doi: 10.1093/hmg/ddg001

39. Evonuk KS, Baker BJ, Doyle RE, Moseley CE, Sestero CM, Johnston $\mathrm{BP}$, et al. Inhibition of system xc- transporter attenuates autoimmune inflammatory demyelination. J Immunol. (2015) 195:450-63. doi: 10.4049/jimmunol.1401108

40. Li G, Yamasaki R, Fang M, Masaki K, Ochi H, Matsushita T, et al. Novel disease-modifying anti-rheumatic drug iguratimod suppresses chronic experimental autoimmune encephalomyelitis by down-regulating activation of macrophages/microglia through an NF-кB pathway. Sci Rep. (2018) 8:1933. doi: 10.1038/s41598-018-20390-5

41. Grishchuk Y, Peña KA, Coblentz J, King VE, Humphrey DM, Wang $\mathrm{SL}$, et al. Impaired myelination and reduced brain ferric iron in the mouse model of mucolipidosis IV. Dis Models Mech. (2015) 8:1591-601. doi: $10.1242 / \mathrm{dmm} .021154$

42. Doeppner TR, Kaltwasser B, Bähr M, Hermann DM. Effects of neural progenitor cells on post-stroke neurological impairment-a detailed and comprehensive analysis of behavioral tests. Front Cell Neurosci. (2014) 8:338. doi: $10.3389 /$ fncel.2014.00338

43. Ramírez-Franco JJ, Munoz-Cuevas FJ, Luján R, Jurado S. Excitatory and inhibitory neurons in the hippocampus exhibit molecularly distinct large dense core vesicles. Front Cell Neurosci. (2016) 10:202. doi: 10.3389/fncel.2016.00202

44. Fernández-Arjona M, Grondona JM, Granados-Durán P, Fernández-Llebrez P, López-Ávalos MD. Microglia morphological categorization in a rat model of neuroinflammation by hierarchical cluster and principal components analysis. Front Cell Neurosci. (2017) 11:235. doi: 10.3389/fncel.2017. 00235

45. Yamasaki R, Fujii T, Wang B, Masaki K, Kido MA, Yoshida M, et al. Allergic inflammation leads to neuropathic pain via glial cell activation. J Neurosci. (2016) 36:11929-45. doi: 10.1523/JNEUROSCI.1981-16.2016

46. Cardona AE, Huang D, Sasse ME, Ransohoff RM. Isolation of murine microglial cells for RNA analysis or flow cytometry. Nat Protocols (2006) 1:1947-51. doi: 10.1038/nprot.2006.327 
47. Katayama Y, Nishiyama M, Shoji H, Ohkawa Y, Kawamura A, Sato T, et al. CHD8 haploinsufficiency results in autistic-like phenotypes in mice. Nature (2016) 537:675. doi: 10.1038/nature19357

48. Subramanian A, Tamayo P, Mootha VK, Mukherjee S, Ebert BL, Gillette MA, et al. Gene set enrichment analysis: a knowledge-based approach for interpreting genome-wide expression profiles. Proc Natl Acad Sci. (2005) 102:15545-50. doi: 10.1073/pnas.0506580102

49. Zabala A, Vazquez-Villoldo N, Rissiek B, Gejo J, Martin A, Palomino A, et al. P2X4 receptor controls microglia activation and favors remyelination in autoimmune encephalitis. EMBO Mol Med. (2018) 2018:e8743. doi: 10.15252/emmm.201708743

50. Tietz SM, Zwahlen M, Haghayegh Jahromi N, Baden P, Lazarevic I, Enzmann $\mathrm{G}$, et al. Refined clinical scoring in comparative EAE studies does not enhance the chance to observe statistically significant differences. Eur J Immunol. (2016) 46:2481-3. doi: 10.1002/eji.201546272

51. Ji N, Kovalovsky A, Fingerle-Rowson G, Guentzel MN, Forsthuber TG. Macrophage migration inhibitory factor promotes resistance to glucocorticoid treatment in EAE. Neurol Neuroinflamm. (2015) 2:e139. doi: 10.1212/NXI.0000000000000139

52. Theis M, Giaume C. Connexin-based intercellular communication and astrocyte heterogeneity. Brain Res. (2012) 1487:88-98. doi: 10.1016/j.brainres.2012.06.045

53. Liddelow SA, Guttenplan KA, Clarke LE, Bennett FC, Bohlen CJ, Schirmer L, et al. Neurotoxic reactive astrocytes are induced by activated microglia. Nature (2017) 541:481-7. doi: 10.1038/nature21029

54. Mizuno T, Doi Y, Mizoguchi H, Jin S, Noda M, Sonobe Y, et al. Interleukin34 selectively enhances the neuroprotective effects of microglia to attenuate oligomeric amyloid- $\beta$ neurotoxicity. Am J Pathol. (2011) 179:2016-27. doi: 10.1016/j.ajpath.2011.06.011

55. Mayo L, Trauger SA, Blain M, Nadeau M, Patel B, Alvarez JI, et al. Regulation of astrocyte activation by glycolipids drives chronic CNS inflammation. Nat Med. (2014) 20:1147-56. doi: 10.1038/nm.3681

56. Rasmussen S, Wang Y, Kivisäkk P, Bronson RT, Meyer M, Imitola $\mathrm{J}$, et al. Persistent activation of microglia is associated with neuronal dysfunction of callosal projecting pathways and multiple sclerosis-like lesions in relapsing-remitting experimental autoimmune encephalomyelitis. Brain (2007) 130:2816-29. doi: 10.1093/brain/awm219

57. Rothwell NJ, Luheshi GN. Interleukin 1 in the brain: biology, pathology and therapeutic target. Trends Neurosci. (2000) 23:618-25. doi: 10.1016/S0166-2236(00)01661-1

58. Liu JS-H, Zhao M-L, Brosnan CF, Lee SC. Expression of inducible nitric oxide synthase and nitrotyrosine in multiple sclerosis lesions. Am J Pathol. (2001) 158:2057-66. doi: 10.1016/S0002-9440(10)64677-9

59. Sharief M, Noori M, Ciardi M, Cirelli A, Thompson E. Increased levels of circulating ICAM-1 in serum and cerebrospinal fluid of patients with active multiple sclerosis. correlation with TNF- $\alpha$ and blood-brain barrier damage. $J$ Neuroimmunol. (1993) 43:15-21. doi: 10.1016/0165-5728(93)90070-F

60. Rieckmann P, Albrecht M, Kitze B, Weber T, Tumani H, Broocks A, et al. Tumor necrosis factor- $\alpha$ messenger RNA expression in patients with relapsing-remitting multiple sclerosis is associated with disease activity. Annal Neurol. (1995) 37:82-8. doi: 10.1002/ana.410370115

61. Kurumbail RG, Kiefer JR, Marnett LJ. Cyclooxygenase enzymes: catalysis and inhibition. Curr Opin Struct Biol. (2001) 11:752-60. doi: 10.1016/S0959-440X(01)00277-9

62. Mokoena T, Gordon S. Human macrophage activation. Modulation of mannosyl, fucosyl receptor activity in vitro by lymphokines, gamma and alpha interferons, and dexamethasone. J Clin Invest. (1985) 75:624-31. doi: 10.1172/JCI111740

63. Stein M, Keshav S, Harris N, Gordon S. Interleukin 4 potently enhances murine macrophage mannose receptor activity: a marker of alternative immunologic macrophage activation. J Exp Med. (1992) 176:287-92. doi: 10.1084/jem.176.1.287

64. Andreou KE, Soto MS, Allen D, Economopoulos V, de Bernardi A, Larkin JR, et al. Anti-inflammatory microglia/macrophages as a potential therapeutic target in brain metastasis. Front Oncol. (2017) 7:251. doi: $10.3389 /$ fonc. 2017.00251
65. Berard JL, Kerr BJ, Johnson HM, David S. Differential expression of SOCS1 in macrophages in relapsing-remitting and chronic EAE and its role in disease severity. Glia (2010) 58:1816-26. doi: 10.1002/glia. 21051

66. Rezvanpour A, Santamaria-Kisiel L, Shaw GS. The S100A10-annexin A2 complex provides a novel asymmetric platform for membrane repair. J Biol Chem. (2011) 286:40174-83. doi: 10.1074/jbc.M111. 244038

67. Li J, Riau AK, Setiawan M, Mehta JS, Ti S-E, Tong L, Tan DT, et al. S100A expression in normal corneal-limbal epithelial cells and ocular surface squamous cell carcinoma tissue. Mol Vis. (2011) 17:2263.

68. Hsu SY, Kaipia A, Zhu L, Hsueh AJ. Interference of BAD (Bcl-xL/Bcl-2-associated death promoter)-induced apoptosis in mammalian cells by 14-3-3 isoforms and P11. Mol Endocrinol. (1997) 11:1858-67.

69. Chen MJ, Kress B, Han X, Moll K, Peng W, Ji RR, et al. Astrocytic CX43 hemichannels and gap junctions play a crucial role in development of chronic neuropathic pain following spinal cord injury. Glia (2012) 60:1660-70. doi: 10.1002/glia.22384

70. Cronin M, Anderson PN, Cook JE, Green CR, Becker DL. Blocking connexin43 expression reduces inflammation and improves functional recovery after spinal cord injury. Mol Cell Neurosci. (2008) 39:152-60. doi: 10.1016/j.mcn.2008.06.005

71. Huang C, Han X, Li X, Lam E, Peng W, Lou N, et al. Critical role of connexin 43 in secondary expansion of traumatic spinal cord injury. J Neurosci. (2012) 32:3333-8. doi: 10.1523/JNEUROSCI.121611.2012

72. Takeuchi H, Jin S, Wang J, Zhang G, Kawanokuchi J, Kuno R, et al. Tumor necrosis factor- $\alpha$ induces neurotoxicity via glutamate release from hemichannels of activated microglia in an autocrine manner. J Biol Chem. (2006) 281:21362-68. doi: 10.1074/jbc.M600504200

73. Hansen DB, Braunstein TH, Nielsen MS, MacAulay N. Distinct permeation profiles of the connexin 30 and 43 hemichannels. FEBS Lett. (2014) 588:144657. doi: 10.1016/j.febslet.2014.01.036

74. Wang Y, Szretter KJ, Vermi W, Gilfillan S, Rossini C, Cella M, et al. IL34 is a tissue-restricted ligand of CSF1R required for the development of Langerhans cells and microglia. Nat Immunol. (2012) 13:753. doi: 10.1038/ ni. 2360

75. Cekanaviciute E, Dietrich HK, Axtell RC, Williams AM, Egusquiza R, Wai KM, et al. Astrocytic TGF- $\beta$ signaling limits inflammation and reduces neuronal damage during central nervous system Toxoplasma infection. J Immunol. (2014) 2014:1303284. doi: 10.4049/jimmunol. 1303284

76. Cekanaviciute E, Fathali N, Doyle KP, Williams AM, Han J, Buckwalter MS. Astrocytic transforming growth factor-beta signaling reduces subacute neuroinflammation after stroke in mice. Glia (2014) 62:1227-40. doi: $10.1002 /$ glia. 22675

Conflict of Interest Statement: RY has received honoraria from Biogen Japan and Japan Blood Products Organization; Takeda Pharmaceutical Co. Ltd., and Mitsubishi Tanabe Pharma; JK is a consultant for Biogen Japan and Medical Review and has received honoraria from Bayer Healthcare, Mitsubishi Tanabe Pharma, Nobelpharma, Otsuka Pharmaceutical, Sanofi K.K., Chugai Pharmaceutical Co. Ltd., Teijin Pharma, Novartis Pharma, and Medical Review.

The remaining authors declare that the research was conducted in the absence of any commercial or financial relationships that could be construed as a potential conflict of interest.

Copyright (c) 2018 Fang, Yamasaki, Li, Masaki, Yamaguchi, Fujita, Isobe and Kira. This is an open-access article distributed under the terms of the Creative Commons Attribution License (CC BY). The use, distribution or reproduction in other forums is permitted, provided the original author(s) and the copyright owner(s) are credited and that the original publication in this journal is cited, in accordance with accepted academic practice. No use, distribution or reproduction is permitted which does not comply with these terms. 